\title{
Flora vascular y vegetación del Cerro El TePOPOte, JALISCO, MÉXICO
}

\author{
Alfredo Frías-Castro, Arturo Castro-Castro1, Jesús Guadalupe González-Gallegos, \\ Esteban Alberto SuÁrez-Muro y Francisco Javier Rendón-Sandoval \\ Instituto de Botánica, Departamento de Botánica y Zoología, Centro Universitario de Ciencias Biológicas y \\ Agropecuarias, Universidad de Guadalajara, Zapopan, Jalisco, México. \\ ${ }^{1}$ Autor para la correspondencia: arca68@hotmail.com
}

\begin{abstract}
Resumen: El cerro El Tepopote se localiza en la Faja Volcánica Transmexicana, en la confluencia de las regiones fisiográficas Cañones, Cuencas Centrales y Montañosa-Declives del Pacífico, al oeste de Guadalajara, Jalisco. La vegetación dominante es el bosque de Quercus y Pinus y su flora se compone de 76 familias, 214 géneros y 370 especies, con numerosos endemismos, algunas novedades para la región y especies nuevas para la ciencia. La diversidad de su flora es mayor que la observada en el Área de Protección de Flora y Fauna La Primavera y el Área de Proteción Hidrológica Bosque El Nixticuil-San Esteban-El Diente, áreas con las que mantiene una alta semejanza florística y geológica. El estado de conservación de El Tepopote es discutido, con base en indicadores ambientales derivados de los resultados florísticos.
\end{abstract}

Palabras clave: bosque El Nixticuil-San Esteban-El Diente, corredor biológico, diversidad florística, endemismo, La Primavera.

\begin{abstract}
El Tepopote hill is located in the west extreme of the Trans-Mexican Volcanic Belt and the confluence of the physiographic regions of the Canyons, Central Basins and Mountainous-Pacific slopes, at western Guadalajara, Jalisco. Oak-pine forest is the dominant vegetation type and the floristic list of the area includes 76 families, 214 genera and 370 species, with numerous endemisms, novelties for the region, and new species for the science. The diversity of its flora is higher than those at the La Primavera Flora and Fauna Protected Area and El Nixticuil-San Esteban-El Diente Hidrologic Protected Area, with which maintains high floristic and geologic similarities. The state of conservation of El Tepopote is discussed, based on environmental indexes derived from the floristic studies.
\end{abstract}

Key words: biological corridor, El Nixticuil-San Esteban-El Diente forest, endemism, floristic diversity, La Primavera.

$\mathbf{L}$ a importancia de documentar la riqueza florística en muchos sitios de México es inapelable considerando la destrucción acelerada de los ecosistemas y el conocimiento incompleto de sus componentes (Rzedowski, 1991; Villaseñor, 1992; Espejo-Serna et al., 2004). Además, siendo las plantas la base de la producción primaria de los ecosistemas y quienes proveen de múltiples servicios ambientales que sustentan el desarrollo humano, es imperioso contar con un conocimiento completo de su riqueza.

El occidente de México es una región que concentra una alta diversidad vegetal (Villaseñor, 1991; Ramírez-Delgadillo et al., 2010). Esta riqueza atrajo a más de 250 colectores botánicos en el periodo de 1790 a 1970 (McVaugh, 1972a). Como muestra, alrededor de 230 taxa fueron descritos a partir de colectas realizadas sólo en algunas de las barrancas y

montañas aledañas a los municipios de Guadalajara y Zapopan en el estado de Jalisco, además de un considerable número de novedades fitogeográficas (McVaugh, 1952, 1972a; Acevedo-Rosas et al., 2008).

El extremo oeste del municipio de Zapopan, Jalisco, es una área de novedades botánicas (p. ej. Ayers, 1987; Solano y Ríos-Gómez, 2011; Castro-Castro et al., 2012). Ésta se localiza justo en el parteaguas de las cuencas de los Ríos Santiago y Ameca, vinculando al Área de Protección de Flora y Fauna La Primavera (APFFLP) y al Área Natural Protegida Bosque El Nixticuil-San Esteban-El Diente (APH BENSEDI) con la barranca del Río Santiago. Es decir, se encuentra a poco más de un kilómetro de distancia del APFFLP y a no más de 14 kilómetros del BENSEDI; no obstante, el área no ha sido considerada parte de alguno de los decretos que delimitan 


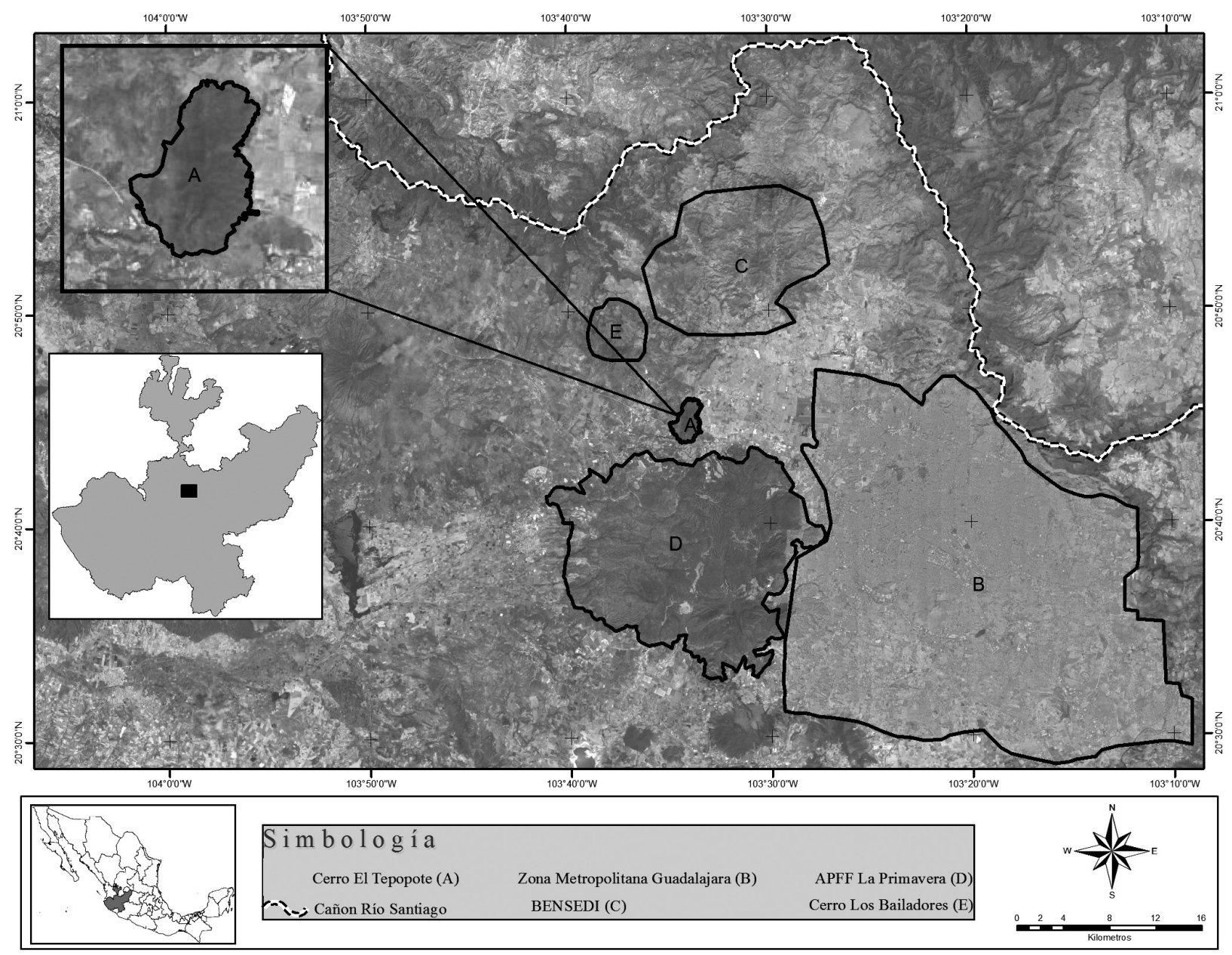

Figura 1. Localización del área de estudio.

tales áreas de protección. Villavicencio-García et al. (2009), determinaron el grado global de conectividad forestal del APFFLP con el extremo oeste de Zapopan, identificando a los Cerros Bailadores y El Tepopote como elementos de enlace entre el APFFLP y la Barranca del Río Santiago, constituyendo un corredor biológico con dirección noreste (Figura 1).

En la actualidad, se ejerce una fuerte presión sobre el bosque por parte de inmobiliarias que pretenden desarrollar fraccionamientos en el cerro El Tepopote. A pesar de que el uso de suelo determinado en el Ordenamiento Ecológico Territorial del municipio de Zapopan, Jalisco, corresponde al de área de protección de flora y fauna, con una política de protección y de fragilidad ambiental media (SEMADES, 2006). En el mismo documento se establece que estas áreas deben ser destinadas a actividades relacionadas con la preservación, repoblación, propagación, aclimatación, refugio, investigación y aprovechamiento sustentable de las especies de flora y fauna, así como las relativas a la educación y difusión.

Ante el desconocimiento biológico y la amenaza que enfrenta el cerro El Tepopote y sus alrededores, los objetivos del trabajo se concentraron en documentar la diversidad florística, las formas biológicas de su flora y el estado de conservación del área. Un propósito adicional fue contrastar la diversidad de su flora con otras áreas que cuentan con un inventario florístico en el estado de Jalisco.

\section{Material y métodos}

Área de estudio. El Tepopote se localiza en el extremo oeste de la zona metropolitana de Guadalajara y al noreste del municipio de Tala, entre las coordenadas extremas $103^{\circ} 33^{\prime}$ a $103^{\circ} 35^{\prime}$ de longitud oeste y $20^{\circ} 43^{\prime}$ a $20^{\circ} 46^{\prime}$ de latitud norte. Tiene una forma ovada que corre de norte a sur, una superficie aproximada de 655 hectáreas y es perpendicular a la orientación tanto de la barranca del Río Santiago como del bosque de La Primavera. Presenta una variación en altitud que va de 1,600 a 1,940 m en su punto más alto. La porción alta exhibe pendientes suaves, de donde se desprenden varias cañadas con pendiente abrupta y suelos más rocosos. Se localiza en la coincidencia de las provincias fisiográficas Faja Volcánica Transmexicana y Sierra Madre Occidental 
(Rossotti et al., 2002) y de acuerdo con Gutiérrez-Vázquez (1959) en la confluencia de tres regiones fisiográficas: de los Cañones, de las Cuencas Centrales y de la MontañosaDeclives del Pacífico. Al sur está delimitado por la carretera Guadalajara-Nogales, al este por campos de cultivo, y al norte y oeste por bosques de Quercus abiertos utilizados para pastoreo (Figura 1).

En el área no ocurren corrientes de agua superficiales permanentes, pero sí se forman varios arroyos durante la época húmeda del año. El clima de la región es templado subhúmedo con lluvias en verano e invierno $\left[\mathrm{S}\left(\mathrm{w}_{1}\right)(\mathrm{w})\right]$ y temperatura media anual de $20.6{ }^{\circ} \mathrm{C}$. El suelo se compone por regosol y litosol. La unidad litológica más característica de la región es la denominada Toba Tala, constituida en su mayor porcentaje por fragmentos de pómez y rocas ígneas extrusivas ácidas.

Trabajo de campo. Comprendió la recolección selectiva de materiales botánicos en el periodo de finales de 2008 a mediados de 2011, mediante recorridos en promedio mensuales y con mayor intensidad en los periodos lluviosos, alcanzando 30 días de exploración. Las muestras vegetales obtenidas se procesaron siguiendo métodos convencionales para la preparación de ejemplares de herbario, descritos por Lot y Chiang (1986) y Sánchez-González y González (2007). La colección principal fue depositada en el herbario IBUG y duplicados fueron distribuidos a los herbarios MEXU, IEB y ENCB (acrónimos de acuerdo a Thiers, 2011).

Trabajo de gabinete. Fueron revisados diversos trabajos florísticos pertinentes para la extracción de la información de los ejemplares correspondientes al área de estudio (McVaugh, 1961，1983，1984，1985，1987，1989, 1992, 1993a, b, 1995, 2001; González-Villarreal, 1986, 1990, 1996; Cervantes-Aceves, 1992; Mickel, 1992). También se examinó meticulosamente la colección del herbario IBUG. Con base en la información obtenida a través del trabajo de campo, la revisión de literatura y de especímenes de herbario, se generó un listado florístico y una base de datos en la que además se incorporó información sobre el estado de conservación, forma biológica por hábito, hábitat y tipo de nutrición, y distribución de los taxa.

Las formas biológicas presentes en las plantas reflejan adaptaciones a las condiciones ecológicas en que se desarrollan (Galán de Mera et al., 1999). De esta manera, la comparación de las proporciones de formas biológicas vegetales presentes entre dos o más sitios, nos provee información sobre las condiciones ecológicas de los mismos (Pausas y Austin, 2001). Esta clase de comparaciones son comunes en listados florísticos por unidades políticas (Vázquez-García et al., 2004; Bloch-Petersen et al., 2006), biológicas o biogeográficas (Batalha y Martins, 2002; Castro-Díez et al., 2003; García-Franco et al., 2008; López-Pérez et al., 2011; León-de La Luz, 2012), por áreas de conservación (Váz-
quez-G. et al., 1995; Meave et al., 2008; Espinosa-Jiménez et al., 2011; Pérez-Farrera et al., 2012) o por sitios puntuales (Wang et al., 2002; Jiménez-Gómez y Lorente-Adame, 2004; Ren et al., 2012); o bien, desde el aspecto evolutivo (Day et al., 1992) o ecológico (Gill y Burke, 1999). Sin embargo, no existe consistencia en el uso de este concepto, en la práctica se utilizan de manera diferente, entre distintas publicaciones, términos tales como forma biológica (LópezPérez et al., 2011; Pérez-Farrera et al., 2012), forma de vida (Day et al., 1992; Gill y Burke, 1999; Batalha y Martins, 2002; Jiménez-Gómez y Lorente-Adame, 2004; Wang et al., 2002; Bhattarai y Vetaas, 2003; Bloch-Petersen et al., 2006; Castro-Díez et al., 2003; García-Franco et al., 2008), y forma de crecimiento (Meave et al., 2008; Espinosa-Jiménez et al., 2011); o aún dentro de una misma publicación (León-de La Luz et al., 2012), para referirse básicamente a lo mismo. Además, la heterogeneidad en que se maneja esta información es amplia; algunos documentos se basan en un sistema de clasificación de fuentes originales (Batahla y Martins, 2002; Bloch-Petersen et al., 2006; López-Pérez et al., 2011; Ren et al., 2012) o secundarias (Espinosa-Jiménez et al., 2011), otros mencionan seguir los criterios de algún autor pero con modificaciones propias (Espinosa-Jiménez et al., 2011; León-de La Luz et al., 2012), y existen también quienes no citan que propuesta es la que están siguiendo (Day et al., 1992; Gill y Burke, 1999; Bhattarai y Vetaas, 2003; Castro-Díez et al., 2003; Jiménez-Gómez y Lorente-Adame, 2004; García-Franco et al., 2008; Meave et al., 2008; Pérez-Farrera et al., 2012). Por otra parte, dentro de los criterios de forma biológica que siguen algunos autores existe una mezcla de características de diferente índole, tales como hábito, forma de nutrición o sustrato en que crece el organismo (p. ej. Galán de Mera et al., 1999; EspinosaJiménez et al., 2011); esto hace que se establezcan categorías que no son mutuamente excluyentes, lo cual dificulta el proceso de asignar taxa a una categoría y generan un sesgo o dificultad en las comparaciones. Halloy (1990) propone un sistema en que se contemplan características de la silueta, hoja, tallo, raíz e inflorescencia de una planta para definir la forma biológica a la que pertenece; sin embargo, la propuesta resulta impráctica de utilizar. Una propuesta que ha sido aceptada y utilizada por varios autores (Batalha y Martins, 2002; Bloch-Petersen et al., 2006; López-Pérez, et al. 2011; Ren et al., 2012) es la de Raunkiaer (1934); empero, se ha cuestionado su aplicabilidad a áreas tropicales debido a que se basa en la posición del meristemo durante el invierno, entendido este último como época desfavorable que limita el crecimiento vegetal, situación que no tiene significado en áreas donde la estacionalidad no es marcada (Sarmiento y Monasterio, 1983). Además, esa misma propuesta también mezcla criterios de diferente índole, ya que las terofitas (una de las categorías incluidas) se definen por un atributo de perennidad y no por la posición del meristemo; engloba hábitos diferentes en una misma categoría, tal es el caso de 
las fanerofitas; en este grupo están incluidos arbustos, árboles y trepadoras perennes. En ocasiones es ambigua la aplicación de sus definiciones, como el caso de las plantas epífitas que bien pueden considerarse como fanerofitas o hemicriptofitas en función de si se considera como punto de referencia al suelo o al hospedero. La heterogeneidad aquí señalada y las inconsistencias de propuestas de forma biológica como la de Raunkiaer, enmarcan la necesidad de una revisión exhaustiva de esta temática; derivado de ello en el presente trabajo se decidió seguir la definición de forma biológica de Font-Quer (1953) y una clasificación provisional que considera categorías distribuidas en tres atributos distintos, en las que quedaron englobadas las plantas del listado aquí obtenido: hábito (árbol, arbusto, bejuco o liana, hierba), hábitat (epífito, rupícola, terrestre) y tipo de nutrición (autótrofa, hemiparásita, holoparásita y saprófita). Fueron utilizadas las definiciones de Moreno (1984) y Hickey y King (2000), y se cotejó con los listados de Vázquez-G. et al. (1995) y Vázquez-García (2004) para reconocer y asignar las plantas del listado a las formas biológicas correspondientes.

La distribución de las especies del área se juzgó con base en una matriz de presencia/ausencia por entidad federativa y un campo en que se agregaron otros países en que han sido registradas; la información de presencia se extrajo a partir de la consulta de la base de datos del Missouri Botanical Garden (Tropicos, 2011). La matriz generada está disponible y puede ser solicitada a los autores.

Las familias, géneros, especies, subespecies y variedades se presentan en orden alfabético bajo los criterios de clasificación propuestos por Mickel y Smith (2004) para helechos, Dahlgren et al. (1985) para monocotiledóneas y APG (2009) para dicotiledóneas en sentido amplio. Los nombres de los géneros y especies se citan de acuerdo con las bases de datos de Tropicos (2011) y la forma de citar a los autores sigue el catálogo de Villaseñor et al. (2008).

Para comparar la riqueza de especies en áreas de diferentes tamaños se utilizó el índice de biodiversidad taxonómica, que es el número de especies registradas dividido por el logaritmo natural del área en $\mathrm{km}^{2}(I B=S / \ln A$, donde $S$ es el número de especies registradas y $A$ el área; Squeo et $a l ., 1998)$. Finalmente, se identificaron especies incluidas en alguna categoría de la Norma Oficial Mexicana de la Secretaría de Medio Ambiente y Recursos Naturales (NOM-059ECOL-2010; SEMARNAT, 2010).

\section{Resultados}

Tipos de vegetación. En el área predomina el bosque de Quercus-Pinus, aunque en determinadas porciones es mayor la presencia del bosque de Quercus abierto con pastizal y también algunos relictos de bosque tropical caducifolio, principalmente en el extremo suroeste. En porciones de pendientes suaves y extensas, la vegetación tiende a ser más uniforme y con menor riqueza de plantas. En contraste, en las cañadas la vegetación es más heterogénea y con mayor riqueza, inclusive con algunas plantas que pueden estar restringidas a una sola cañada, como es el caso de Aralia humilis, Campyloneurum phyllitidis, Epidendrum anisatum, Escobedia grandiflora, Govenia superba, Habenaria mariae, Magnolia pugana, Moussonia elegans, Quercus conspersa, Schoenocaulon jaliscense var. jaliscense, Sedum jaliscanum, Solanum stoloniferum y Viola oxyodontis.

Los manchones de bosque de Quercus-Pinus y Quercus abiertos con pastizal se entremezclan, haciendo difícil una delimitación clara de los mismos. En ambos, el estrato arbóreo está dominado por Quercus castanea, Q. magnoliifolia, $Q$. resinosa y Pinus oocarpa. Por otra parte, $Q$. castanea, $Q$. coccolobifolia, $Q$. conspersa y $Q$. rugosa se restringen a la parte más alta, a partir de $1,800 \mathrm{~m}$ s.n.m. y $Q$. viminea se restringe a las cañadas. También es frecuente encontrar Agarista mexicana var. pinetorum, Bejaria aestuans, Clethra rosei, Comarostaphylis glaucescens, Diphysa suberosa, Ficus petiolaris y F. velutina. La mayoría de los árboles tienen en promedio $6 \mathrm{~m}$ de alto y alcanzan como máximo $10(-12) \mathrm{m}$ de altura. En el estrato arbustivo predominan las compuestas y leguminosas, siendo frecuentes Ageratum corymbosum, Baccharis salicifolia, Bouvardia terniflora, Calea ternifolia, Cosmos landii var. achalconensis, Critoniopsis foliosa, C. grandiflora, Desmodium jaliscanum, Styrax jaliscana, Vaccinium stenophyllum y Ximenia parviflora. El estrato herbáceo es el más rico, donde es común observar Aspicarpa brevipes, Anemia jaliscana, Begonia tapatia, Bidens rostrata, Bletia ensifolia, B. roezlii, Brickellia cuspidata, Coreopsis cuneifolia, Desmodium angustifolium, Euphorbia sphaerorhiza, Gibasis linearis subsp. rhodantha, Iostephane heterophylla, Ipomoea capillacea, Macrosiphonia hypoleuca, Prochnyanthes mexicana, Psacalium poculiferum, Roldana sessilifolia, Salvia angustiarum, S. firma y Stevia viscida. Las epífitas están representadas por tres especies de helechos: Campyloneurum phylliditis, Phlebodium areolatum y Polypodium furfuraceum; las hemiparásitas por Buchnera obliqua, Castilleja arvensis, Escobedia grandiflora y Lamourouxia viscosa, las holoparásitas sólo por Lennoa madreporoides y las saprófitas por Hexalectris brevicaulis.

En las porciones de bosque tropical caducifolio el estrato arbóreo presenta una altura de 4-8 $\mathrm{m}$, pero Leucaena macrophylla y Lysiloma acapulcense alcanzan hasta 10-12 m de altura. Los árboles más frecuentes son Acaciella angustissima var. angustissima, A. tequilana, Bursera fagaroides, B. multijuga, B. penicillata, Colubrina greggii, Ficus pertusa, Tecoma stans y Lippia umbellata. Entre los arbustos de afinidad tropical destacan Asterohyptis stellulata, Buddleia sessiliflora, Bunchosia palmeri, Diphysa suberosa, D. thurberi, Hyptis albida, Kosteletzkya tubiflora, Mimosa albida var. strigosa, Montanoa karvinskii y Triumfetta polyandra. Las hierbas más comunes son Asclepias contrayerba, Bidens 
odorata, Chamaecrista absus var. meonandra, C. nictitans var. jaliscensis, C. serpens var. wrightii, Ipomoea stans, Lasiacis nigra, Loeselia mexicana, Salvia tiliifolia y Sida linifolia. También están presentes de manera frecuente las trepadoras Galium mexicanum, Clematis dioica, Dictyanthus sepicola, Ipomoea laeta, I. orizabensis y Toxicodendron radicans. Las epífitas y hemiparásitas están representadas exclusivamente por Phoradendron bolleanum.

Diversidad florística. Los trabajos de campo y gabinete comprendieron la recolección de 410 ejemplares y la consulta de 277 exsicata depositadas en el herbario IBUG. Como resultado, se elaboró un listado de la flora vascular compuesto por 370 especies, 214 géneros y 76 familias, donde están representadas cuatro divisiones (Apéndice 1; Cuadro 1). La división Magnoliophyta es la más diversa con 337 taxa, seguida de Polypodiophyta (30 spp.), Pinophyta (2 spp.) y Lycopodiophyta (1 sp.). Dentro de las angiospermas, las clases Liliopsida y Magnoliopsida registraron 84 y 253 especies respectivamente. Las familias mejor representadas son Asteraceae (64 especies, 17\%), Fabaceae (57, 15\%), Poaceae $(37,9.9 \%)$, Orchidaceae (20, $5.3 \%)$, Pteridaceae $(16,4.3 \%)$, Fagaceae $(11,3 \%)$, Lamiaceae $(10,3 \%)$, Solanaceae $(8,2 \%)$, Apocynaceae, Euphorbiaceae y Malvaceae (7, 1.9\% c/u), Agavaceae y Apiaceae $(6,1.6 \% \mathrm{c} / \mathrm{u})$. En estas familias se concentra $70 \%$ de la diversidad total del área.

Los géneros más diversos fueron: Muhlenbergia (12 especies); Desmodium y Quercus (10 c/u); Cheilanthes, Dalea y Salvia (6 c/u); Asclepias, Bletia, Euphorbia, Habenaria, Malaxis y Solanum (5 c/u); Adiantum, Ageratina, Bursera, Chamaecrista, Cuphea, Eryngium, Ficus, Mimosa, Stevia, Verbesina y Viguiera $(4 \mathrm{c} / \mathrm{u})$.

De acuerdo con el índice de diversidad taxonómica, el área de estudio presenta una riqueza florística de 196 especies por $\mathrm{km}^{2}$. Una comparación con otras áreas de bosque de Quercus y Pinus en Jalisco permite estimar que la riqueza florística del cerro El Tepopote es elevada, aunque esta apreciación debe tomarse con cautela debido a las diferencias en el esfuerzo de muestreo entre autores, los criterios para delimitar comunidades y la impresición en el cálculo de las superficies (Cuadro 2).

Cuadro 1. Diversidad florística por grupos taxonómicos encontrada en el cerro El Tepopote, municipio de Zapopan, Jalisco, México.

\begin{tabular}{lccccc}
\hline Grupo & Familias & Géneros & Especies & Variedades Subespecies \\
\hline Lycopodiophyta & 1 & 1 & 1 & - & - \\
Polypodiophyta & 9 & 18 & 30 & 3 & - \\
Pinophyta & 1 & 1 & 2 & - & - \\
Magnoliophyta & 65 & 194 & 337 & 20 & 2 \\
Total & 76 & 214 & 370 & 23 & 2 \\
\hline
\end{tabular}

Forma biológica de la flora por hábito, hábitat y tipo de nutrición. El hábito representado por una mayor cantidad de especies en el área es el de las hierbas (258 especies, $70 \%)$, después arbustos $(53,14 \%)$, árboles $(40,11 \%)$, y bejucos $(19,5 \%)$. Las plantas dominantes, según el tipo de hábitat que ocupan, son las terrestres $(359,97 \%)$, mientras las rupícolas $(7,2 \%)$ y epífitas $(4,1 \%)$ son por igual escasas. De acuerdo al tipo de nutrición las plantas autótrofas son las más abundantes en el área (363, 98\%), luego las hemiparásitas $(5,1 \%)$, saprófitas y holoparásitas $(1,1 \%$ en conjunto, Apéndice 1).

Distribución geográfica y endemismo de las especies. El cerro El Tepopote, comparte más del $45 \%$ de su flora con seis estados de México: Nayarit, Michoacán, Estado de México, Oaxaca, Guerrero y Chiapas (Cuadro 3). En otro contexto, más de la mitad de su flora se comparte con al menos otro país americano $(185,54 \%)$, y el resto corresponde a especies endémicas del país. En el área destaca la presencia de especies y variedades endémicas de Jalisco, como Coreopsis cyclocarpa, Cosmos landii var. achalconensis, Habenaria mariae, Perityle jaliscana, Polianthes zapopanensis, Schoenocaulon jaliscense var. jaliscense, Stevia ovalis y Wedelia grayi (Hernández-López, 1995). Otras especies presentan una distribución restringida al occidente mexicano, como Agave guadalajarana, Baccharis occidentalis, Brickellia cuspidata, Coreopsis cuneifolia, Magnolia pugana, Mammillaria jaliscana, Manfreda involuta, Phaseolus jaliscanus, Salvia angustiarum, S. firma, S. heterotricha, Sedum jaliscanum, Styrax jaliscana, Verbesina tequilana, Viguiera palmeri y V. schultzii. Algunas especies distribuidas principalmente en la Sierra Madre Occidental alcanzan su límite de distribución sur en el occidente de Jalisco, como Quercus conspersa y $Q$. praineana (González-Villarreal, 1986).

Especies importantes para la conservación. Producto de la revisión y colecta del material botánico para el presente estudio, fue el descubrimiento y descripción de una especie nueva del género Dahlia Cav. (Castro-Castro et al., 2012) y una especie nueva del género Muhlenbergia Schreb. (en preparación). Hasta ahora, estos dos taxa sólo se conocen del cerro El Tepopote, en un rango altitudinal entre 1,800 y 1,950 m y en cañadas húmedas de exposición noreste, en el bosque mixto de Quercus y Pinus. La superficie reducida en que se encuentran y la presión inmobiliaria a que está sujeta el área, incrementan el riesgo de estos taxa a la extinción. Por otro lado, en El Tepopote crecen Selaginella porphyrospora, Campyloneurum phyllitidis y Schoenocaulon jaliscense var. jaliscense, tres especies listadas en la NOM059-SEMARNAT-2010 bajo las categorías: en peligro de extinción, amenazada y bajo protección especial, respectivamente (SEMARNAT, 2010; Apéndice 1). 


\section{Alfredo Frías-Castro et al.}

Cuadro 2. Comparación de la riqueza florística del cerro El Tepopote, municipio de Zapopan, Jalisco con otras áreas que cuentan con inventarios florísticos en el estado, utilizando el índice de diversidad taxonómica. APFFLP = Área de Protección de Flora y Fauna La Primavera. APHBENSEDI = Área de Proteción Hidrológica Bosque El Nixticuil-San Esteban-El Diente. $\ln A=\operatorname{logaritmo}$ natural del área en km². Abreviaturas: F = Familias; $\mathrm{G}=$ Géneros; $\mathrm{S}=$ Especies; ND = No determinado.

Tipos de vegetación de acuerdo a Rzedowski y McVaugh (1966) y Rzedowski (1978). Abreviaturas: BQ = bosque de Quercus; BES = bosque espinoso; $\mathrm{BG}=$ bosque de galería; $\mathrm{BA}=$ bosque de Abies; $\mathrm{BNM}=$ bosque mesófilo de montaña; $\mathrm{BP}=$ bosque de Pinus; $\mathrm{BPQ}=$ bosque de Pinus y Quercus; $\mathrm{BTC}=$ bosque tropical caducifolio; $\mathrm{BTS}=$ bosque tropical subcaducifolio; $\mathrm{MX}=$ matorral xerófilo; $\mathrm{P}=$ pastizal; $\mathrm{VA}=$ vegetación acuática; $\mathrm{VH}=$ vegetación halófila; $\mathrm{VS}$ = vegetación secundaria; VSA = vegetación sabanoide.

\begin{tabular}{|c|c|c|c|c|c|c|c|c|c|}
\hline Fuente & Localidad & $\begin{array}{l}\text { Área } \\
\left(\mathrm{km}^{2}\right)\end{array}$ & $\begin{array}{l}\text { Rango } \\
\text { altitudinal }\end{array}$ & $\begin{array}{l}\text { Tipos de } \\
\text { vegetación }\end{array}$ & $\begin{array}{l}\text { Número de } \\
\text { exploraciones }\end{array}$ & $\mathrm{F}$ & G & E & $\begin{array}{r}\text { Riqueza } \\
(\mathrm{sp} / \ln A)\end{array}$ \\
\hline SEMARNAT, 2000 & APFFLP & 305 & $1,800-2,200$ & $\mathrm{BTC}, \mathrm{BPQ}, \mathrm{BQ}, \mathrm{VA}$ & ND & 107 & 419 & 805 & 140 \\
\hline Barba-Robert, 2001 & Ferrería de Tula & 45.93 & $2,200-2,860$ & $B P, B P Q, B G, V S, P, V A$ & 56 & 93 & 289 & 524 & 137 \\
\hline $\begin{array}{l}\text { Contreras-Rodríguez } \\
\text { et al., } 2000\end{array}$ & Piedras Bola & ND & $1,500-2,300$ & BTC, BG, P, BQ & ND & 43 & 99 & 139 & ND \\
\hline Cortés-Romero, 2000 & $\begin{array}{l}\text { Laguna de } \\
\text { Cajititlán }\end{array}$ & 74.1 & $1,500-1,800$ & BTC, BES, VA, VS & 46 & 82 & 285 & 469 & 109 \\
\hline García-Rubio, 2003 & Papas de Arriba & 15 & 2,260 & $M X, B Q, P, V A, V S$ & 23 & 65 & 220 & 339 & 125 \\
\hline Guerrero-Nuño, 1994 & Sierra de Quila & 320 & $1,300-2,560$ & $\begin{array}{l}\text { BES, BTC, BQ, BPQ, } \\
\text { BMM, BG, }\end{array}$ & 45 & 128 & 427 & 772 & 134 \\
\hline $\begin{array}{l}\text { Guerrero-Hernández, } \\
2012\end{array}$ & Juanacatlán & 3.97 & $2,120-2,420$ & $\mathrm{BMM}, \mathrm{BA}, \mathrm{VS}$ & 36 & 84 & 195 & 289 & 210 \\
\hline Harker et al., 2008 & Papas de Arriba & 25 & 2,260 & $M X, P$ & ND & 73 & 230 & 356 & 110 \\
\hline $\begin{array}{l}\text { Hernández-Toro, } \\
2003\end{array}$ & $\begin{array}{l}\text { Tecolotlán y } \\
\text { María García }\end{array}$ & 850 & $0-920$ & $\begin{array}{l}\text { BTS, BTC, BES, P, BQ, } \\
\text { VA, VSA }\end{array}$ & 250 & 129 & 493 & 1,029 & 152 \\
\hline Lott, 1993 & Bahía de Chamela & 350 & $0-500$ & BTC, BTS, VH & ND & 124 & 544 & 1,120 & 191 \\
\hline $\begin{array}{l}\text { Machuca-Nuñez, } \\
1989\end{array}$ & Cerro Viejo & 100 & $1,900-2,300$ & $\mathrm{BM}, \mathrm{BP}, \mathrm{BPQ}, \mathrm{BTC}, \mathrm{P}, \mathrm{VA}$ & 97 & 162 & 511 & 990 & 214 \\
\hline $\begin{array}{l}\text { Macías-Rodríguez y } \\
\text { Ramírez-Delgadillo, } \\
2000\end{array}$ & Cerro del Colli & 3.5 & $1,700-1,950$ & $\mathrm{BQ}, \mathrm{BTC}, \mathrm{VS}$ & 5 & 53 & 132 & 188 & 150 \\
\hline $\begin{array}{l}\text { Nieves-Hernández, } \\
2002\end{array}$ & Norte de Jalisco & ND & $400-2,800$ & $\begin{array}{l}\mathrm{BPQ}, \mathrm{BP}, \mathrm{BQ}, \mathrm{BTC}, \mathrm{BMM}, \\
\mathrm{MX}, \mathrm{BTC}, \mathrm{P}\end{array}$ & ND & 132 & 676 & 1,652 & ND \\
\hline $\begin{array}{l}\text { Ramírez-Delgadillo } \\
\text { et al., } 2006\end{array}$ & APH-BENSEDI & 159 & $1,550-1,620$ & BTC, BQ, BPQ, BES, P, VS & ND & 77 & 225 & 456 & 90 \\
\hline $\begin{array}{l}\text { Rodríguez y Reynoso- } \\
\text { Dueñas, } 1992\end{array}$ & $\begin{array}{l}\text { Bosque Escuela } \\
\text { La Primavera }\end{array}$ & 6.72 & $1,390-1,700$ & $\mathrm{BPQ}, \mathrm{BTC}, \mathrm{VA}, \mathrm{VS}$ & 48 & 73 & 213 & 323 & 169 \\
\hline $\begin{array}{l}\text { Vázquez-G. et al., } \\
1995\end{array}$ & $\begin{array}{l}\text { Sierra de } \\
\text { Manantlán }\end{array}$ & 13,960 & $400-2,800$ & $\begin{array}{l}\text { BMM, BA, BPQ, BQ, BP, MX, } \\
\text { BTC, BES, BTS, VG, P, VSA }\end{array}$ & ND & 181 & 981 & 2,774 & 291 \\
\hline $\begin{array}{l}\text { Vázquez-García } \\
\text { et al., } 2004\end{array}$ & Norte de Jalisco & ND & $800-2,800$ & $\begin{array}{l}\text { BPQ, BTC, BMM, MX, P, } \\
\text { VA, BES }\end{array}$ & ND & 151 & 732 & 2,079 & ND \\
\hline Villegas-Flores, 1993 & Laguna de Sayula & 27 & 1,300 & VA, BES, BTC, VH, VS & ND & 76 & 208 & 306 & 93 \\
\hline $\begin{array}{l}\text { Wynter-Warra et al., } \\
2003\end{array}$ & Cerro Gordo & 5.68 & $2,400-2,600$ & BQ, BTC, BG, VS & ND & 64 & 184 & 278 & 160 \\
\hline Este trabajo & Cerro El Tepopote & 6.55 & $1,600-1,940$ & $\mathrm{BQ}, \mathrm{BPQ}$ & 30 & 76 & 214 & 370 & 196 \\
\hline
\end{tabular}




\section{Discusión}

Este trabajo representa el primer esfuerzo para obtener un listado florístico, que caracteriza la vegetación y presenta un diagnóstico de la flora del cerro El Tepopote. El área de estudio alberga 32\% de las familias, 13\% de los géneros y 6\% de las especies registradas para Jalisco (RamírezDelgadillo et al., 2010). Rzedowski (1991) considera que alrededor del $50 \%$ de la flora mexicana es endémica al territorio del país, siendo las regiones montañosas cubiertas por bosques de Quercus y Pinus un centro importante de diversificación y endemismo vegetal; una situación similar ocurre en El Tepopote donde la mayor parte de la vegetación corresponde a bosque de Quercus y Pinus y el $45 \%$ de sus especies son endémicas de México. En el caso particular de Jalisco, Hernández-López (1995) coincide en señalar a los bosques de Quercus y Pinus como los más ricos en cantidad de endemismos.

Entre las especies endémicas que crecen en El Tepopote, se identifican diez elementos que se restringen a Jalisco (Hernández-López, 1995), mientras que Dahlia pugana y Muhlenbergia sp. nov. ined. hasta ahora se conocen sólo del municipio de Zapopan (Apéndice 1).

Algunos de los géneros con mayor cantidad de especies encontrados en el Tepopote corresponden con los resultados de otros trabajos florísticos realizados en la región y en vegetaciones semejantes, estos son Quercus, Desmodium, Salvia y Muhlenbergia (Guerrero-Nuño, 1994; Vázquez-G. et al., 1995; Barba-Robert, 2001; Nieves-Hernández, 2002; Wynter-Warra et al., 2003; Guerrero-Hernández, 2012), además están incluidos dentro de los 20 géneros más diversos señalados para el país por Villaseñor (2004), y difieren con aquellos de sitios con una mayor proporción de vegetación de afinidad tropical (Hernández-Toro, 2003) o xerofítica (García-Rubio, 2003). No es fortuito que la mayor riqueza se concentre en estos géneros, ya que México ha fungido como centro de diversificación y alberga una riqueza considerable de los mismos (McVaugh, 1972b; 1987; Ramamoorthy y Elliott, 1998; Dávila-Aranda et al., 2004; Herrera-Arrieta y Peterson, 2007).

El Tepopote presentó una cifra mayor en el índice de diversidad taxonómica que el calculado para 11 de los 19

Cuadro 3. Especies registradas en el cerro El Tepopote, municipio de Zapopan, Jalisco y compartidas con otros estados de México.

\begin{tabular}{lc}
\hline Estado & Número de especies compartidas \\
\hline Nayarit & $211(62 \%)$ \\
Michoacán & $200(58 \%)$ \\
Estado de México & $182(54 \%)$ \\
Oaxaca & $181(53 \%)$ \\
Guerrero & $167(49 \%)$ \\
Chiapas & $166(49 \%)$ \\
\hline
\end{tabular}

sitios en Jalisco que cuentan con un listado florístico a la fecha (Rodríguez y Reynoso-Dueñas, 1992; Lott, 1993; Villegas-Flores, 1995; Guerrero-Nuño, 1994; SEMARNAT, 2000; Cortés-Romero, 2000; Macías-Rodríguez y RamírezDelgadillo, 2000; Wynter-Warra et al., 2003; Barba-Robert, 2001; García-Rubio, 2003; Hernández-Toro, 2003; Ramírez-Delgadillo et al., 2006; Harker et al., 2008; Cuadro 2). Cabe señalar que en cinco de los trabajos incluidos en el Cuadro 2 no es posible obtener un índice de diversidad debido a la carencia de datos sobre las dimensiones del área de trabajo. Por otro lado, los sitios que obtuvieron menor índice de diversidad taxonómica son heterogéneos entre sí en cuanto a su superficie, la vegetación que los compone y el rango altidutidinal que abarcan. Entre ellos, destaca el trabajo de Hernández-Toro (2003) en los municipios de Tomatlán y Cabo Corrientes, ya que en él se incluye un área mayor, aborda más variedad de tipos de vegetación y muestra predominancia de elementos tropicales; sin embargo, registra un valor menor de especies por $\mathrm{km}^{2}$, que sólo se puede explicar por insuficiencia en el esfuerzo de muestreo en función de las dimensiones del área. Aunque como se señaló, estos valores se deben considerar con mesura.

En contraste, los trabajos de Machuca-Núñez (1989), Vázquez-G. et al. (1995) y Guerrero-Hernández (2012) exhiben los mayores valores de diversidad taxonómica con respecto a los trabajos realizados en Jalisco. Este resultado no es del todo sorprendente y no se explica en función de la intensidad de muestreo, sino por las características intrínsecas de las mismas áreas. Este es el caso de Manantlán (Vázquez-G. et al., 1995), que se reconoce como una región de alta biodiversidad; además de ser una de las zonas mejor exploradas, no sólo en un contexto regional sino nacional (Arriaga et al., 2000; CONABIO, 2010). En el caso de Machuca-Núñez (1989) y Guerrero-Hernández (2012), destaca el esfuerzo de muestreo; en estos documentos es posible deducir que al menos cada exploración cubrió cerca de 10 y 1 hectáreas, respectivamente. También vale destacar que se trata de sitios ligados a cuerpos de agua permanentes (lagos de Chapala y Juanacatlán) que sostienen una humedad ambiental elevada, lo que puede influir de forma positiva en el incremento de la riqueza de especies leñosas (Bhattarai y Vetaas, 2003).

Al comparar el presente estudio con el realizado por Rodríguez y Reynoso-Dueñas (1992), que resulta similar en dimensión, variación altitudinal, tipos de vegetación y que además forma parte del mismo sistema orográfico, se puede observar que El Tepopote muestra una mayor riqueza y un índice de diversidad más elevado (Cuadro 2). Por otra parte, la intensidad de muestreo en Rodríguez y Reynoso-Dueñas es poco más elevada que la aplicada en El Tepopote, por lo que aludir a un sesgo en dicha intensidad para explicar las diferencias en riqueza y diversidad no es adecuado. Un factor que podría ser importante es el del grado de perturbación en cada uno de los sitios. En Bosque Escuela La Primavera 
la vegetación está fragmentada por brechas amplias lo que permite el libre acceso al área y un mayor impacto por visitantes, a diferencia de El Tepopote, en donde los accesos son límitados y la influencia antropogénica aún es reducida.

Además de las diferencias en riqueza de especies por unidad de área, también destaca el registro de especies presentes en el cerro El Tepopote y no incluidas en los trabajos florísticos de esos sitios (SEMARNAT, 2000; Ramírez-Delgadillo et al., 2006). Tal es el caso de Epidendrum anisatum, Habenaria mariae, Malaxis myurus, Manfreda involuta, Muhlenbergia scoparia, Polypogon elongatus, Quercus conspersa, Setaria pumila, Sorghastrum incompletum y Trisetum mexicanum, entre otras.

La mayor riqueza florística del área estudiada, con respecto a otros bosques de la Faja Volcánica Transmexicana (Torres-Zúñiga y Tejero-Díez 1998; Cedano-Maldonado y Harker 2000; Wynter-Warra et al., 2003; Medina-Lemus y Tejero-Díez, 2006), probablemente se deba a una historia geológica compleja, en términos de la presencia de una continua actividad volcánica y tectónica como señalan Rossotti et al. (2002) y como producto de la transición entre dos provincias florísticas (Rzedowski, 1978).

En cuanto a las formas biológicas encontradas por hábito, hábitat y tipo de nutrición, hay coincidencia con otros trabajos florísticos realizados en México respecto a la predominancia de las hierbas y plantas autótrofas, y escasez de bejucos (Villegas-Flores, 1995; Barba-Robert, 2001; Nieves-Hernández, 2002; Wynter-Warra et al., 2003; García-Rubio et al., 2003; García-Franco et al., 2008; Guerrero-Hernández, 2012; Espinosa-Jiménez et al., 2011; León-de La Luz et al., 2012; Pérez-Farrera et al., 2012). Contrasta con las proporciones que se mantienen en áreas de bosque tropical perennifolio como la estudiada en el Monumento Natural Yaxchilán, Chiapas (Meave et al., 2008), donde los árboles son el hábito dominante. Es probable que la alta proporción de hierbas respecto al resto de hábitos encontrados sea producto de las características intrínsecas de la dinámica de este gremio: tiempos generacionales cortos, capacidad rápida de desplazamiento o dispersión y suceptibilidad a factores locales tales como las características de suelo, grado de disturbio y cobertura del dosel que proveen una variedad de nichos disponibles; lo anterior sugerido por resultados obtenidos en un análisis de la variación de riqueza en gradientes altitudinales (Bhattarai y Vetaas, 2003).

En términos de conservación regional, la riqueza observada es relevante dado que en un área pequeña se concentra una importante cantidad de especies de la flora del estado de Jalisco, que incluye elementos endémicos, especies protegidas por las normas nacionales, así como especies nuevas para la ciencia. En este sentido, uno de los criterios más importantes para justificar la conservación de una región es la diversidad de especies y la rareza de los taxa, en la idea de que una diversidad elevada significa mayor calidad ecológi- ca. Por lo anterior se considera que la zona de estudio debe considerarse como área prioritaria para la conservación.

\section{Agradecimientos}

Agradecemos al personal del Herbario IBUG las facilidades otorgadas para la consulta, a los siguientes especialistas por la determinación de materiales: Mollie Harker (Asteraceae), Luz María González Villarreal (Fagaceae, Ericaceae y Clethraceae), Raymundo Ramírez-Delgadillo $†$ (Fabaceae) y Aarón Rodríguez Contreras (Solanaceae y monocotiledóneas petaloides). Así mismo, agradecemos a Blanca O. Ávila Ramírez, Sara I. González Castro y José Luis Villalpando por la determinación de muestras de la familia Poaceae. A Sergio Zamudio y un revisor anónimo por las importantes sugerencias hechas al manuscrito. Gracias a Guadalupe Munguía-Lino, Virginia Ramírez-Cruz, Ernesto De CastroArce y a Jesús Padilla-Lepe por su ayuda durante el trabajo de campo. Un agradecimiento especial a Luis Felipe Bugarin-Navarro (Geoservicios) por el decidido apoyo en la elaboración de la cartografía.

\section{Literatura citada}

Acevedo-Rosas R., Hernández-Galaviz M. y Cházaro-Basáñez M. 2008. Especies de plantas vasculares descritas de las barrancas aledañas a la ciudad de Guadalajara y de Río Blanco, Jalisco, México. Polibotánica 26:1-38.

APG [The Angiosperm Phylogeny Group]. 2009. An update of the Angiosperm Phylogeny Group classification for the orders and families of flowering plants: APG III. Botanical Journal of the Linnean Society 161:106-121.

Arriaga L., Espinoza J.M., Aguilar C., Martínez E., Gómez L. y Loa E. Coords. 2000. Regiones terrestres prioritarias de México. Comisión Nacional para el Conocimiento y uso de la Biodiversidad. México, D.F.

Ayers T.J. 1987. Four species from western Mexico new to Lobelia (Campanulaceae: Lobelioideae). Brittonia 39:417-422.

Barba-Robert E.M. 2001. Florística de la cuenca presa Ferrería de Tula y zonas aledañas en el municipio de Tapalpa, Jalisco. Tesis profesional. Centro Universitario de Ciencias Biológicas y Agropecuarias, Universidad de Guadalajara, Guadalajara. 94 pp.

Batalha M.A. y Martins R. M. 2002. Life-form spectra of Brazilian cerrado sites. Flora - Morphology, Distribution, Functional Ecology of Plants 197:452-460.

Bhattarai K.R. y Vetaas O.R. 2003. Variation in plant species richness of different life forms along a subptropical elevation gradient in the Himalayas, east Nepal. Global Ecology \& Biogeography 12:327-340.

Bloch-Petersen M., Brandt J. y Olsen M. 2006. Integration of European habitat monitoring based on plant life form composition as an indicator of environmental change and change in biodiversity. Danish Journal of Geography 106:61-74.

Castro-Castro A., Rodríguez A., Vargas-Amado G. y Harker M. 2012. Diversidad del género Dahlia (Asteraceae: Coreopsideae) en Jalisco, México y descripción de una especie nueva. Revista Mexicana de Biodiversidad 83:347-358. 
Castro-Díez P., Montserrat-Martí G. y Cornelissen J.H.C. 2003. Trade-offs between phenology, relative growth rate, life form and seed mass among 22 Mediterraneaen woody species. Plant Ecology 166:117-129.

Cedano-Maldonado M. y Harker M. 2000. Listado florístico preliminar del Volcán Ceboruco, Nayarit, México. Boletín del Instituto de Botánica 8:137-168.

Cervantes-Aceves N. 1992. La familia Malvaceae en el estado de Jalisco, México. Colección Flora de Jalisco 3. Instituto de Botánica, Universidad de Guadalajara. Guadalajara.

CONABIO [Comisión Nacional para el Conocimiento y Uso de la Biodiversidad]. 2010. El Bosque Mesófilo de Montaña en México: Amenazas y Oportunidades para su Conservación y Manejo Sostenible. CONABIO. México, D.F.

Contreras-Rodríguez S.H., Romo-Campos R.L. y Reynoso-Dueñas J.J. 2000. Caracterización de la vegetación en la zona de Piedras Bola, Ahualulco de Mercado, Jalisco, México. Boletín del Instituto de Botánica 7:103-121.

Cortés-Romero C. 2000. Florística de la región de Cajititlán, municipio de Tlajomulco de Zúñiga, Jalisco, México. Tesis de licenciatura. Centro Universitario de Ciencias Biológicas y Agropecuarias, Universidad de Guadalajara, Guadalajara. 89 pp.

Dahlgren R.M.T., Clifford H.T. y Yeo P.F. 1985. The Families of Monocotyledons. Structure, Evolution and Taxonomy. Springer-Verlag. Berlín.

Dávila-Aranda P., Lira-Saade R. y Valdés-Reyna J. 2004. Endemic species of grasses in Mexico: a phytogeographic approach. Biodiversity and Conservation 13:1101-1121.

Day T.A., Vogelmann T.C. y DeLucia E.H. 1992. Are some plant life forms more effective than others in screening out ultraviolet-B radiation? Oecologia 92:513-519.

Espejo-Serna A., López-Ferrari A.R. y Salgado-Ugarte I. 2004. A current estimate of angiosperm diversity in Mexico. Taxon 53:127-130.

Espinosa-Jiménez J.A., Pérez-Farrera M.A. y Martínez-Camilo R. 2011. Inventario florístico del parque Nacional Cañón del Sumidero, Chiapas, México. Boletín de la Sociedad Botánica de México 89:37-82.

Font-Quer P. 1953. Diccionario de Botánica. Editorial Labor. Barcelona.

Galán de Mera A., Hagen M.A. y Vicente-Orellana J.A. 1999. Aerophyte, A new life form in Raunkiaer's classification? Journal of Vegetation Science 10:65-68.

García-Franco J.G., Castillo-Campos G., Mehltreter K., Martínez M.L. y Vázquez G. 2008. Composición florística de un bosque mesófilo del centro de Veracruz, México. Boletín de la Sociedad Botánica de México 83:37-52.

García-Rubio L.A. 2003. Listado florístico del rancho Las Papas de Arriba, municipio de Ojuelos de Jalisco, México. Tesis profesional. Centro Universitario de Ciencias Biológicas y Agropecuarias, Universidad de Guadalajara, Guadalajara. 66 pp.

Gill R.A. y Burke I.C. 1999. Ecosystem consequences of plant life form changes at three sites in the semiarid United States. Oecologia 121:551-563.

González-Villarreal L.M. 1986. Contribución al conocimiento del género Quercus (Fagaceae) en el estado de Jalisco. Colección Flora de Jalisco 1. Instituto de Botánica, Universidad de Guadalajara, Guadalajara.

González-Villarreal L.M. 1990. Las Ericáceas de Jalisco, México. Colección Flora de Jalisco 2. Instituto de Botánica, Universidad de Guadalajara, Guadalajara.

González-Villarreal L.M. 1996. La familia Clethraceae en el estado de Jalisco, México. Colección Flora de Jalisco 5. Instituto de Botánica, Universidad de Guadalajara, Guadalajara.

Guerrero-Hernández R. 2012. Flora y vegetación asociada a Abies guatemalensis var. jaliscana Martínez en Juanacatlán, municipio de Mascota, Jalisco. Tesis profesional. Centro Universitario de Ciencias Biológicas y Agropecuarias, Universidad de Guadalajara, Guadalajara. 62 pp.

Guerrero-Nuño J.J. 1994. Contribución al conocimiento de la vegetación y la flora de la Sierra de Quila, Jal., Mex. Tesis de licenciatura. Universidad de Guadalajara. 73 pp.

Gutiérrez-Vázquez M. 1959. Geografía física de Jalisco. Tesis de maestría. Facultad de Filosofía y Letras, Universidad Nacional Autónoma de México, México D.F. 133 pp.

Halloy S. 1990. A morphological classification of plants, with special reference to the New Zealand alpine flora. Journal of Vegetation Science 1:291-304.

Harker M., García-Rubio L.A. y Riojas-López M.E. 2008. Composición florística de cuatro hábitats en el rancho Las Papas de Arriba, Municipio de Ojuelos de Jalisco, Jalisco, México. Acta Botanica Mexicana 85:1-29.

Hernández-Toro I.M. 2003. Flora y vegetación de entre los ríos Tecolotlán y María García, municipios de Cabo Corrientes y Tomatlán, Jalisco. Tesis de doctorado. Universidad de Salamanca, Salamanca. $521 \mathrm{pp}$.

Hernández-López L. 1995. The endemic flora of Jalisco, Mexico, centers of endemism and implications for conservation. Tesis de maestría, University of Wisconsin. Madison. 76 pp.

Herrera-Arrieta Y. y Peterson P.M. 2007. Muhlenbergia (Poaceae) de Chihuahua, México. SIDA, Botanical Miscellany 29:1-109.

Hickey M. y King C. 2000. The Cambridge Illustrated Glossary of Botanical Terms. Cambridge University Press., Cambridge.

Jiménez-Gómez R.G. y Lorente-Adame R.G. 2004. Fenología y especies acompañantes de la milpilla perenne (Zea diploperennis Iltis, Doebley y Guzmán). En: Cuevas-Guzmán R. y JardelPeláes E.J. Eds. Flora y Vegetación de la Estación Científica Las Joyas, pp. 205-228, Universidad de Guadalajara, Guadalajara.

León-de la Luz J.L., Domínguez-Cadena R. y Medel-Narváez A. 2012. Florística de la selva baja caducifolia de la Península de Baja California, México. Botanical Sciences 90:143-162.

López-Pérez Y., Tejero-Díez J.D., Torres-Díaz A.N. y Luna-Vega I. 2011. Flora del bosque mesófilo de montaña y vegetación adyacente en Avándaro, Valle de Bravo, Estado de México, México. Boletín de la Sociedad Botánica de México 88:35-53.

Lot A. y Chiang F. 1986. Manual de Herbario. Administración y Manejo de Colecciones, Técnicas de Recolección y Preparación de Ejemplares Botánicos. Consejo Nacional de la Flora de México, A.C. México, D.F.

Lott E.J. 1993. Annotated checklist of the vascular flora of the Chamela Bay Region, Jalisco, Mexico. Occasional Papers of the California Academy of Sciences 148:1-60.

Machuca-Núñez J.A. 1989. Florística y ecología de la vegetación fanerogámica de la región septentrional de Jocotepec, Jalisco (México). Tesis profesional. Centro Universitario de Ciencias Biológicas y Agropecuarias, Universidad de Guadalajara, Guadalajara. 221 pp.

Macías-Rodríguez M.A. y Ramírez-Delgadillo R. 2000. Florística del Cerro del Colli, municipio de Zapopan, Jalisco, México. Boletín del Instituto de Botánica 8:75-99. 
McVaugh R. 1952. The barranca of Guadalajara and its place in the botanical literature. The Asa Gray Bulletin 1:385-390.

McVaugh R. 1961. Euphorbiaceae novae Novo-Galicianae. Brittonia 13:145-205.

McVaugh R. 1972a. Botanical exploration in Nueva Galicia, Mexico, from 1790 to the present time. Contributions from the University of Michigan Herbarium 9:205-357.

McVaugh R. 1972b. Flora Novo-Galiciana (Quercus). Contributions from the University of Michigan Herbarium 12:1-93.

McVaugh R. 1983. Flora Novo-Galiciana (Gramineae) XIV. University of Michigan Press, Ann Arbor.

McVaugh R. 1984. Flora Novo-Galiciana (Compositae) XII. University of Michigan Press, Ann Arbor.

McVaugh R. 1985. Flora Novo-Galiciana (Orchidaceae) XVI. University of Michigan Press, Ann Arbor.

McVaugh R. 1987. Flora Novo-Galiciana (Leguminosae) V. University of Michigan Press, Ann Arbor.

McVaugh R. 1989. Flora Novo-Galiciana (Bromeliaceae to Dioscoreaceae) XV. University of Michigan Press, Ann Arbor.

McVaugh R. 1992. Flora Novo-Galiciana (Gymnospems to Pteridophytes) XVII. University of Michigan Press, Ann Arbor.

McVaugh R. 1993a. Flora Novo-Galiciana (Limnocharitaceae to Typhaceae) XIII. University of Michigan Press, Ann Arbor.

McVaugh R. 1993b. Euphorbiacearum sertum Novo-Galicianarum Revisarum. Contributions from the University of Michigan Herbarium 19:207-239.

McVaugh R. 1995. Euphorbiacearum sertum Novo-Galicianarum revisarum. Contributions from the University of Michigan Herbarium 20:173-215.

McVaugh R. 2001. Flora Novo-Galiciana (Ochnaceae to Loasaceae) III. University of Michigan Press, Ann Arbor.

Meave J.A., Romero-Romero M.A., Valle-Doménech A., RincónGutiérrez A., Martínez E. y Ramos C.H. 2008. Plant diversity assessment in the Yaxchilán Natural Monument, Chiapas, Mexico. Boletín de la Sociedad Botánica de México 83:53-76.

Medina-Lemus J.G. y Tejero-Díez J.D. 2006. Flora y vegetación del Parque Estatal Atizapán-Valle Escondido, Estado de México, México. Polibotánica 21:1-43.

Mickel J.T. 1992. Pteridophytes and fern allies. En: McVaugh R. Ed. Flora Novo-Galiciana (Gymnosperms and Pteridophytes). University of Michigan Press, Ann Arbor.

Mickel J.T. y Smith A.R. 2004. The Pteridophytes of Mexico. New York Botanical Garden Press, Nueva York.

Moreno N.P. 1984. Glosario Botánico Ilustrado. Instituto Nacional de Investigaciones sobre Recursos Bióticos (CECSA), Xalapa.

Nieves-Hernández G. 2002. Flora vascular del norte de Jalisco y su uso tradicional por la etnia huichola, Jalisco, México. Tesis de maestría. Centro Universitario de Ciencias Biológicas y Agropecuarias, Universidad de Guadalajara, Guadalajara. 152 pp.

Pausas J.G. y Austin M.P. 2001. Patterns of plant species richness in relation to different environments: an appraisal. Journal of Vegetation Science 12:153-166.

Pérez-Farrera M.A., Martínez-Camilo R., Martínez-Meléndez N., Farrera-Sarmiento O. y Maza-Villalobos S. 2012. Listado florístico del Cerro Quetzal (Polígono III) de la Reserva de la Biosfera El Triunfo, Chiapas, México. Botanical Sciences 90:113-142.

Ramamoorthy T.P. y Elliott M. 1998. Lamiaceae de México: diversidad, distribución, endemismo y evolución. En: Ramamoorthy T.P., Bye R., Lot A. y Fah J. Eds. Diversidad Biológica de México. Orígenes y Distribución, pp. 501-526, Instituto de Biología,
Universidad Nacional Autónoma de México, México, D.F.

Ramírez-Delgadillo R, Reynoso-Dueñas J.J., Castro-Castro A., Mercado-Muñoz F. y Frías-Castro A. 2006. Flora y vegetación. En: Loza-Llamas A. Coord. Estudio técnico justificativo para declarar área natural protegida el área Bosque El Nixticuil-San Esteban-El Diente del municipio de Zapopan. Universidad de Guadalajara-H. Ayuntamiento de Zapopan, Jalisco. <nixticuil. blogspot.com/2007/11/estudios-tcnicos-justificativos-para. html> (consultado 20 febrero 2012)

Ramírez-Delgadillo R., Vargas-Ponce O., Arreola-Nava H.J., Cedano-Maldonado M., González-Tamayo R., González-Villarreal L.M., Harker M., Hernández-López L., Martínez-González R.E., Pérez de la Rosa J.A., Rodríguez-Contreras A., ReynosoDueñas J.J., Villarreal de Puga L.M. y Villaseñor J.L. 2010. Catálogo de Plantas Vasculares de Jalisco. Universidad de Guadalajara/Sociedad Botánica de México/Universidad Autónoma Metropolitana, Guadalajara.

Raunkiaer C. 1934. The Life Forms of Plants and Statistical Plant Geography. Oxford University Press. Oxford.

Ren X., Yang G., Zhu F., Qin X., Wang D., Liu Z. y Feng Y. 2012. Plant communities, species richness and life-forms along elevational gradients in Taibai Mountain, China. African Journal of Agricultural Research 7:1834-1848.

Rodríguez A. y Reynoso-Dueñas J.J. 1992. Inventario florístico del Bosque-Escuela, Sierra de La Primavera, Municipio de Tala, Jalisco, México. Boletin del Instituto de Botánica 1:137-166.

Rossotti A., Ferrari L., López-Martínez M. y Rosas-Elguera J. 2002. Geology of the boundary between the Sierra Madre Occidental and the Trans-Mexican Volcanic Belt in the Guadalajara region, western Mexico. Revista Mexicana de Ciencias Geológicas 19:1-15.

Rzedowski J.1978. Vegetación de México. Limusa. México, D.F.

Rzedowski J. 1991. Diversidad y orígenes de la flora fanerogámica de México. Acta Botanica Mexicana 14:3-21.

Rzedowski J. y McVaugh R. 1966. La vegetación de Nueva Galicia. Contributions from the University of Michigan Herbarium 9:1-123.

Sánchez-González A. y González L.M. 2007. Técnicas de recolecta de plantas y herborización. En: Contreras-Ramos A., CuevasCardona C., Goyenechea I. e Iturbide U. Eds. La Sistemática, Base del Conocimiento de la Biodiversidad. Universidad Autónoma del Estado de Hidalgo, Pachuca.

Sarmiento G. y Monasterio M. 1983. Life forms and phenology. En: Bourliere F.E. Ed. Ecosystems of the World XIII. Tropical Savannas, pp. 79-108, Elsevier, Amsterdam.

SEMARNAT [Secretaría de Medio Ambiente y Recursos Naturales y Comisión Nacional de Áreas Naturales Protegidas]. 2000. Programa de Manejo Área de Protección de Flora y Fauna La Primavera. SEMARNAT. México, D.F.

SEMARNAT. 2010. Norma Oficial Mexicana NOM-059-ECOL2010, Protección ambiental-Especies nativas de México de flora y fauna silvestres-Categorías de riesgo y especificaciones para su inclusión, exclusión o cambio-Lista de especies en riesgo. Diario Oficial de la Federación. 2a Sección, 30 de diciembre de 2010

SEMADES [Secretaría de Medio Ambiente para el Desarrollo Sustentable] 2006. Modelo de ordenamiento ecológico territorial del estado de Jalisco. SMADS.<siga.jalisco.gob.mx/moet> (consultado 23 de junio 2011).

Solano E. y Ríos-Gómez R. 2011. Polianthes zapopanensis (Agava- 
ceae), una especie nueva de Jalisco, México. Brittonia 63:70-74.

Squeo F.A., Cavieres L.A., Arancio G., Novoa J. E., Matthei O., Marticorena C., Rodríguez R., Arroyo M.T.K. y Muñoz M. 1998. Biodiversidad de la flora vascular en la Región de Antofagasta, Chile. Revista Chilena de Historia Natural 71:571-591.

Thiers B. 2011. Index Herbariorum: A global directory of public herbaria and associated staff. New York Botanical Garden's Virtual Herbarium.<sweetgum.nybg.org/ih/> (consultado 22 de junio 2011).

Torres-Zúñiga M.M. y Tejero-Díez J.D. 1998. Flora y vegetación de la Sierra de Sultepec, Estado de México. Anales del Instituto de Biología, Serie Botánica 69:135-174.

Tropicos. 2011. Missouri Botanical Garden. <Tropicos.org> (consultado 3-7 de agosto 2011, 18 de junio de 2012).

Vázquez-G. J.A., Cuevas-G. R., Cochrane T.S. e Iltis H.H. 1995. Flora de Manantlán: plantas vasculares de la Reserva de la Biosfera Sierra de Manantlán, Jalisco-Colima, México. Sida, Botanical Miscellany 13. Universidad de Guadalajara. Guadalajara.

Vázquez-García J.A., Cházaro-B M.J., Nieves-H. G., Vargas-Rodríguez Y.L., Vázquez-G. M. y Flores-M. A. 2004. Flora del Norte de Jalisco y Etnobotánica Huichola. Universidad de Guadalajara, Guadalajara.

Villaseñor J.L. 1991. Las Heliantheae endémicas a México: una guía hacia la conservación. Acta Botanica Mexicana 15:29-46.

Villaseñor J.L. 1992. Los parques nacionales y otras áreas protegi- das y su papel en la conservación de la riqueza florística. Boletín del Instituto de Botánica de la Universidad de Guadalajara 1:119-130.

Villaseñor J.L. 2004. Los géneros de las plantas vasculares de la flora de México. Boletín de la Sociedad Botánica de México 75:105-135.

Villaseñor J. L., Ortiz E. y Redonda-Martínez, R. 2008. Catálogo de Autores de Plantas Vasculares de México. Universidad Nacional Autónoma de México/Comisión Nacional para el Conocimiento y uso de la Biodiversidad, México, D. F.

Villavicencio-García R., Martínez de Toda S.S., Santiago-Pérez A.L. y Chávez-Hernández A. 2009. La conectividad forestal de las áreas naturales protegidas del estado de Jalisco con otros ambientes naturales. Scientia-CUCBA 11:43-50.

Villegas-Flores E. 1995. Vegetación de la laguna de Sayula. Tesis profesional. Centro Universitario de Ciencias Biológicas y Agropecuarias, Universidad de Guadalajara, Guadalajara. 53 pp.

Wang G., Zhou G., Yang L. y Li Z. 2002. Distribution, species diversity and life-form spectra of plant communities along and altitudinal gradient in the northern slopes of Qiaanshan Mountains, Gansu, China. Plant Ecology 165:169-181.

Wynter-Warra L.E., Reynoso-D. J.J., Ramírez-D. R. y Portillo-M. L. 2003. Flora y vegetación del Cerro Gordo, Jalisco, México. Boletín del Instituto de Botánica 9:47-78.

Recibido: 27 de febrero de 2012

Aceptado: 16 de septiembre de 2012 
Apéndice 1. Catálogo de la flora vascular del Cerro El Tepopote, municipio de Zapopan, Jalisco, México.

Colectores (Cols.): ACC (Arturo Castro-Castro), AFC (Alfredo Frías-Castro), ARC (Aarón Rodríguez-Contreras), CAT (C. AguirreTinoco), CAW (Carlos Anaya-Wihman), CGR (C. González-Rivas), CLDL (Carlos Luis Díaz-Luna), CSR (C. Sánchez-R.), DG (I. García), DJ (David Jimeno), DMS (David M. Spooner), DRS (D. Rodríguez-S.), EASM (Esteban Alberto Suarez-Muro), EEG (E. Espinoza-G.), FMA (F. Medina-Álvarez), FJRS (Francisco Javier Rendón-Sandoval), GNH (Gregorio Nieves-Hernández), GL (Gutierrez-L.), JAG (J. A. García), JAPR (Jorge Alberto Pérez de la Rosa), JBG (J. Becerra-Gómez), JEV (J. E. Villalobos), JGG (Jesús González-Gallegos), JJG (J. J. García-P.), JRR (Jerzy Rzedowski-Rotter), LMVP (Luz María Villarreal de Puga), MC (M. Castrejón), MHS (Mollie Harker-Shumway), MJCB (Miguel de Jesús Cházaro-Bazañes), PAF (Paul A. Fryxell), PFS (P. F. Stevens), RGT (Roberto González-Tamayo), RG (Rafaél Guzmán), RMA (R. M. Araiza), RRM (Ricardo Ramírez-Maciel), SJC (S. J. Cruz), SRV (Salvador Rosillo de Velasco), SZQ (S. Zavala-Quintero).

Forma biológica. Hábito (A): a (árbol), r (arbusto), b (bejuco o liana), h (hierba). Hábitat (H): E (epífito), R (rupícola), T (terrestre). Tipo de nutrición (N): A (autótrofa), M (hemiparásita), L (holoparásita), S (saprófita).

Categoría de riesgo NOM-059-SEMARNAT-2010 (NOM): ANE (amenazada no endémica), PNE (peligro de extinción no endémica), ENE (protección especial no endémica). Símbolos: especie nueva $\left({ }^{*}\right)$, endémica para Jalisco $(†)$.

\begin{tabular}{|c|c|c|c|c|}
\hline A & Especies & Cols./números de colecta & $\mathrm{H} / \mathrm{N}$ & NOM \\
\hline \multicolumn{5}{|c|}{ LYCOPODIOPHYTA } \\
\hline h & $\begin{array}{l}\text { Selaginella porphyrospora A.Braun } \\
\text { POLYPODIOPHYTA } \\
\text { Anemiaceae }\end{array}$ & LMVP 1349 & $\mathrm{R} / \mathrm{A}$ & PNE \\
\hline $\mathbf{h}$ & Anemia jaliscana Maxon & LMVP 2105, 12627 & T/A & \\
\hline $\mathbf{h}$ & $\begin{array}{l}\text { A. tomentosa (Savigny) Sw. var. mexicana (Presl) Mickel } \\
\text { Blechnaceae }\end{array}$ & DG 4951 & T/A & \\
\hline $\mathbf{h}$ & Blechnum glandulosum Kaulf. ex Link & LMVP 2161 & T/A & \\
\hline $\mathbf{h}$ & $\begin{array}{l}\text { Woodwardia spinulosa M.Martens \& Galeotti } \\
\text { Davalliaceae }\end{array}$ & ARC 26; LMVP 2242 & T/A & \\
\hline $\mathbf{h}$ & $\begin{array}{l}\text { Nephrolepis occidentalis Kunze } \\
\text { Dennstaedtiaceae }\end{array}$ & FJRS et al. 529 & T/A & \\
\hline $\mathbf{h}$ & $\begin{array}{l}\text { Pteridium aquilinum (L.) Kuhn var. caudatum (L.) Maxon } \\
\text { Dryopteridaceae }\end{array}$ & DG 4967; LMVP 1507 & T/A & \\
\hline $\mathbf{h}$ & Dryopteris rossii C.Chr. & $\begin{array}{l}\text { ACC } 2124 \text { \& JGG; FJRS et al. 530; } \\
\text { LMVP 2241, } 2460\end{array}$ & T/A & \\
\hline & Ophioglossaceae & & & \\
\hline $\mathbf{h}$ & $\begin{array}{l}\text { Ophioglossum reticulatum } \mathrm{L} \text {. } \\
\text { Polypodiaceae }\end{array}$ & LMVP 599 & T/A & \\
\hline $\mathbf{h}$ & Campyloneurum phyllitidis (L.) Presl & DG 4957 & E/A & ANE \\
\hline $\mathbf{h}$ & Phlebodium areolatum (Humb. \& Bonpl. ex Willd) J.Sm. & $\begin{array}{l}\text { ACC } 2142 \text { \& AFC; ACC } 2116 \text { \& JGG; } \\
\text { FJRS et al. } 532\end{array}$ & E/A & \\
\hline $\mathbf{h}$ & $\begin{array}{l}\text { Polypodium furfuraceum Schltdl. \& Cham. } \\
\text { Pteridaceae }\end{array}$ & DG 4962 & E/A & \\
\hline $\mathbf{h}$ & Adiantum braunii Mett. ex Kuhn & ARC 63 & T/A & \\
\hline $\mathbf{h}$ & A. concinnum Humb. \& Bonpl. ex Willd. & $\begin{array}{l}\text { DG 30; EEG 122; } \\
\text { LMVP 2447; MC s.n. }\end{array}$ & T/A & \\
\hline $\mathbf{h}$ & A. patens Willd. & LMVP 2070 & T/A & \\
\hline $\mathbf{h}$ & A. poiretii Wikstr. & LMVP 2448 & T/A & \\
\hline $\mathbf{h}$ & $\begin{array}{l}\text { Astrolepis aff. integerrima (Hook) D.M.Benham } \\
\text { \& Windman }\end{array}$ & FJRS et al. 534 & T/A & \\
\hline h & A. sinuata (Lag. ex Sw.) D.M.Benham \& Windham & DG 4956; LMVP 4094 & T/A & \\
\hline h & Bommeria pedata (Sw.) Fourn. & LMVP 4604; MC s.n. & T/A & \\
\hline $\mathbf{h}$ & Cheilanthes angustifolia Kunth & DG 4949; FJRS et al. 531 & T/A & \\
\hline $\mathbf{h}$ & C. bonariensis (Willd.) Proctor & LMVP 2262 & T/A & \\
\hline $\mathbf{h}$ & C. farinosa (Forssk.) Kaulf. & EEG 5454 & T/A & \\
\hline
\end{tabular}


Apéndice 1. Continuación

\begin{tabular}{|c|c|c|c|c|}
\hline A & Especies & Cols./números de colecta & $\mathrm{H} / \mathrm{N}$ & NOM \\
\hline $\mathbf{h}$ & C. kaulfussii Kunze & $\begin{array}{l}\text { ACC } 2145 \text { \& AFC; EEG 119; JAG 119; } \\
\text { MC } 119\end{array}$ & T/A & \\
\hline $\mathbf{h}$ & $\begin{array}{l}\text { C. lozanoi (Maxon) R.M.Tryon \& A.F.Tryon } \\
\text { var. seemanii (Hook.) Mickel \& Beitel }\end{array}$ & DG 4948 & $\mathbf{T} / \mathbf{A}$ & \\
\hline h & C. membranacea (Davenp.) Maxon & MC s.n. & T/A & \\
\hline $\mathbf{h}$ & Notholaena brachypus (Kunze) J.Sm & DG 3/4970; JAG 126 & R/A & \\
\hline $\mathbf{h}$ & Pellaea terniflora (Cav.) Link & $\begin{array}{l}\text { ACC } 2149 \text { \& AFC; ACC } 2123 \text { \& JGG; } \\
\text { FJRS et al. } 533\end{array}$ & $\mathbf{R} / \mathbf{A}$ & \\
\hline $\mathbf{h}$ & $\begin{array}{l}\text { Pityrogramma tartarea (Cav.) Maxon } \\
\text { Thelypteridaceae }\end{array}$ & LMVP 2243, 4099 & T/A & \\
\hline $\mathbf{h}$ & Thelypteris interrupta (Willd.) Iwatsuki & DG 4954 & T/A & \\
\hline $\mathbf{h}$ & T. pilosa (M.Martens \& Galeotti) Crawford & LMVP 2459 & T/A & \\
\hline $\mathbf{h}$ & $\begin{array}{l}\text { T. rudis (Kunze) Proctor } \\
\text { PINOPHYTA } \\
\text { PINOPSIDA } \\
\text { Pinaceae }\end{array}$ & DG 4968 & T/A & \\
\hline $\mathbf{a}$ & Pinus devoniana Lindl. & CAW s. n. & T/A & \\
\hline a & $\begin{array}{l}\text { P. oocarpa Schiede ex Schltdl. } \\
\text { MAGNOLIOPHYTA } \\
\text { LILIOPSIDA } \\
\text { Agavaceae }\end{array}$ & FMA s. n. & T/A & \\
\hline $\mathbf{h}$ & Agave guadalajarana Trel. & RMA \& DJ 50 & T/A & \\
\hline $\mathbf{h}$ & Manfreda involuta McVaugh & ACC 2086 \& JGG & T/A & \\
\hline h & M. jaliscana Rose & ACC 2330 \& JGG; AFC 1811 \& JGG & T/A & \\
\hline $\mathbf{h}$ & M. scabra (Ortega) McVaugh & JGG et al. 895 & T/A & \\
\hline $\mathbf{h}$ & Polianthes zapopanensis E.Solano \& Ríos-Gómez † & $\begin{array}{l}\text { ACC } 1840 \text { \& ARC; AFC et al. 1780; } \\
\text { ARC } 5737\end{array}$ & T/A & \\
\hline $\mathbf{h}$ & $\begin{array}{l}\text { Prochnyanthes mexicana (Zucc.) Rose } \\
\text { Anthericaceae }\end{array}$ & ACC 1879 \& AFC; ACC et al. 2187 & T/A & \\
\hline $\mathbf{h}$ & Echeandia flexuosa Greenm. & $\begin{array}{l}\text { ACC } 1890 \text { \& AFC; AFC } 1834 \text { \& JGG; } \\
\text { ARC 5736, 5737; LMVP } 8281\end{array}$ & T/A & \\
\hline & Bromeliaceae & & & \\
\hline $\mathbf{h}$ & Pitcairnia karwinskyana Schult. \& Schult f. & ACC $1849 \&$ ARC & $\mathbf{R} / \mathbf{A}$ & \\
\hline $\mathbf{h}$ & $\begin{array}{l}\text { P. palmeri S.Watson } \\
\text { Calochortaceae }\end{array}$ & ACC et al. 2201 & $\mathbf{R} / \mathbf{A}$ & \\
\hline $\mathbf{h}$ & Calochortus purpureus (Kunth) Baker & $\begin{array}{l}\text { ACC } 1844 \text { \& ARC; AFC et al. 1758, } \\
\text { JGG } 1096\end{array}$ & T/A & \\
\hline & Commelinaceae & & & \\
\hline $\mathbf{h}$ & Commelina coelestis Willd. & $\begin{array}{l}\text { ACC et al. } 2197 ; \text { AFC } 1856 \text { \& JGG., } \\
\text { AFC et al. } 1777\end{array}$ & T/A & \\
\hline $\mathbf{h}$ & $\begin{array}{l}\text { Gibasis linearis (Benth.) Rohw. subsp. rhodantha } \\
\text { (Torr.) D.R.Hunt }\end{array}$ & ACC 2084 \& JGG & T/A & \\
\hline $\mathbf{h}$ & $\begin{array}{l}\text { Tradescantia crassifolia Cav. } \\
\text { Cyperaceae }\end{array}$ & JGG 1094 & T/A & \\
\hline $\mathbf{h}$ & Cyperus esculentus L. & ACC et al. 2198 & T/A & \\
\hline $\mathbf{h}$ & C. flavicomus Michx. & AFC et al. 1768 & T/A & \\
\hline $\mathbf{h}$ & C. sesleriodes Kunth & AFC et al. 1885 & T/A & \\
\hline $\mathbf{h}$ & Eleocharis minima Kunth & AFC et al. 1810 & T/A & \\
\hline
\end{tabular}


Apéndice 1. Continuación

\begin{tabular}{|c|c|c|c|c|}
\hline A & Especies & Cols./números de colecta & $\mathrm{H} / \mathrm{N}$ & NOM \\
\hline h & $\begin{array}{l}\text { Killinga odorata Vahl. } \\
\text { Dioscoreaceae }\end{array}$ & ACC 2090 \& JGG; ACC et al. 2199 & T/A & \\
\hline b & Dioscorea convolvulacea Schltdl. \& Cham. & AFC 1770 et al. & T/A & \\
\hline b & $\begin{array}{l}\text { D. jaliscana S.Watson } \\
\text { Hypoxidaceae }\end{array}$ & LMVP 4603 & T/A & \\
\hline h & $\begin{array}{l}\text { Hypoxis fibrata Brackett } \\
\text { Iridaceae }\end{array}$ & JGG 1092 & T/A & \\
\hline h & Nemastylis tenuis (Herb.) S.Watson & AFC et al. 1872 & T/A & \\
\hline h & Sisyrinchium palmeri Greenm. & ACC 2095 \& JGG & T/A & \\
\hline h & S. pringlei B.L.Rob. \& Greenm. & AFC 1835 \& JGG & T/A & \\
\hline h & $\begin{array}{l}\text { Tigridia dugesii S.Watson } \\
\text { Melanthiaceae }\end{array}$ & ACC 1843 \& ARC; ACC 2091 \& JGG & T/A & \\
\hline h & $\begin{array}{l}\text { Schoenocaulon jaliscense Greenm. var. jaliscense † } \\
\text { Orchidaceae }\end{array}$ & ACC 2111 \& JGG & T/A & ENE \\
\hline h & Bletia adenocarpa Rchb.f. & CAT \& MJCB 6; JGG 1087 & T/A & \\
\hline h & B. ensifolia L.O.Williams & ACC 2099 \& JGG; AFC et al. 1776 & T/A & \\
\hline $\mathbf{h}$ & B. punctata La Llave \& Lex. & ACC 2120a, $2169 \&$ JGG & $\mathrm{T} / \mathrm{A}$ & \\
\hline h & B. reflexa Lindl. & LMVP 2442 & T/A & \\
\hline h & B. roezlii Rchb.f. & ACC 1845 \& ARC; ACC 1877 \& AFC & T/A & \\
\hline h & Epidendrum anisatum La Llave \& Lex. & ACC et al. 2205 & T/A & \\
\hline h & Govenia lagenophora Lindl. & ACC 2168 \& JGG & T/A & \\
\hline h & Habenaria diffusa A.Rich. \& Galeotti & AFC et al. 1836, 1876a & T/A & \\
\hline h & H. jaliscana S.Watson & AFC et al. 1772, 1867; JGG 1088 & T/A & \\
\hline h & H. mariae R.González \& Cuevas-Figueroa† & ACC et al. 2178 & T/A & \\
\hline $\mathbf{h}$ & H. sp. 1 & AFC et al. 1749 & T/A & \\
\hline h & H. sp. 2 & AFC et al. 1785 & T/A & \\
\hline h & Hexalectris brevicaulis L.O.Williams & ACC $1841 \&$ ARC; AFC 1756 \& EASM & $\mathbf{T} / \mathbf{S}$ & \\
\hline h & Liparis vexillifera (La Llave ex Lex.) Cogn. & AFC et al. 1882 & $\mathbf{T} / \mathbf{A}$ & \\
\hline h & Malaxis myurus (Lindl.) Kuntze & SRV \& RGT s. n. & T/A & \\
\hline h & M. soulei L.O.Williams & JGG 1089 & T/A & \\
\hline h & M. unifolia Michx. & ACC et al. 2204 & T/A & \\
\hline h & M. sp. 1 & AFC et al. 1754 & T/A & \\
\hline h & M. sp. 2 & AFC et al. $1754 \mathrm{a}$ & $\mathbf{T} / \mathbf{A}$ & \\
\hline h & M. sp. 3 & AFC et al. 1829 & T/A & \\
\hline & Poaceae & & & \\
\hline h & Aristida jorullensis Kunth & AFC 1785 \& JGG, PFS 1907 & T/A & \\
\hline h & A. sp. 1 & ACC $2132 \&$ AFC & $\mathrm{T} / \mathrm{A}$ & \\
\hline h & Arundinella hispida (Humb. \& Bonpl. ex Willd.) Kuntze & LMVP 7103 & T/A & \\
\hline h & Bouteloua polymorpha (E.Fourn.) Columbus & ACC 2120 \& JGG & T/A & \\
\hline h & B. radicosa (Fourn.) Griffiths & PFS 1906 & T/A & \\
\hline h & Chaetium bromoides (Presl.) Benth. ex Hemsl. & JEV s.n. & T/A & \\
\hline h & Deschampsia sp. & JJGP s.n. & T/A & \\
\hline h & Eragrostis pectinacea (Michx.) Nees & ACC $1871 \&$ AFC & T/A & \\
\hline h & Imperata brevifolia Vasey & LMVP 1525, 4917 & T/A & \\
\hline h & Lasiacis nigra Davidse & ACC 2352 \& JGG ; JRR 14254 & T/A & \\
\hline $\mathbf{h}$ & Muhlenbergia ciliata (Kunth) Trin. & AFC $1837 \&$ JGG & $\mathrm{T} / \mathrm{A}$ & \\
\hline h & M. distichophylla (J.Presl) Kunth & AFC 1826 \& JGG & T/A & \\
\hline h & M. dumosa Scribn. ex Vasey & ACC $2133 \&$ AFC & T/A & \\
\hline h & M. emersleyi Vasey & ACC 2355 \& JGG & T/A & \\
\hline h & M. macroura (Kunth) Hitchc. & LMVP 4704 & T/A & \\
\hline
\end{tabular}


Apéndice 1. Continuación

\begin{tabular}{|c|c|c|c|c|}
\hline A & Especies & Cols./números de colecta & $\mathbf{H} / \mathrm{N}$ & NOM \\
\hline $\mathbf{h}$ & M. robusta (Fourn.) Hitchc. & ACC 2354, 2337 \& JGG; AFC 1827 \& JGG & T/A & \\
\hline $\mathbf{h}$ & M. scoparia Vasey & ACC et al. 2324 & T/A & \\
\hline $\mathbf{h}$ & M. stricta (J.Presl) Kunth & ACC $2130 \&$ AFC & T/A & \\
\hline $\mathbf{h}$ & M. sp. 1 & AFC 1726 \& JGG & T/A & \\
\hline $\mathbf{h}$ & M. sp. 2 & AFC $1727 \&$ JGG & T/A & \\
\hline $\mathbf{h}$ & M. sp. $3^{*+}$ & ACC 2337 \& JGG; ACC 2344 \& JGG & T/A & \\
\hline $\mathbf{h}$ & Oplismenus burmannii (Retz.) P.Beauv. & ACC 2353 \& JGG & T/A & \\
\hline $\mathbf{h}$ & Panicum bulbosum Kunth & ACC $2160 \&$ JGG & T/A & \\
\hline $\mathbf{h}$ & Paspalum humboldtianum Nash & AFC 1820, $2348 \&$ JGG & $\mathbf{T} / \mathbf{A}$ & \\
\hline $\mathbf{h}$ & Polypogon elongatus Kunth & RG 940 & T/A & \\
\hline $\mathbf{h}$ & Schizachirium brevifolium (Sw.) Nees ex Büse & AFC 1831 \& JGG & T/A & \\
\hline $\mathbf{h}$ & S. sanguineum (Retz.) Alston & $\begin{array}{l}\text { ACC } 1883 \text { \& AFC; ACC } 2343 \text { \& JGG; } \\
\text { LMVP } 1331\end{array}$ & T/A & \\
\hline $\mathbf{h}$ & S. tenerum Nees & ACC $2131 \&$ AFC & T/A & \\
\hline $\mathbf{h}$ & Setaria pumila (Poir) Roem & ACC et al. 2188 & T/A & \\
\hline $\mathbf{h}$ & Sorghastrum incompletum (Pers.) Nash & LMVP 12632, 5083 & T/A & \\
\hline $\mathbf{h}$ & Sporobolus macrospermum Scribn. ex Beal. & AFC et al. 1875 & T/A & \\
\hline $\mathbf{h}$ & Trachypogon spicatus (L. f.) Kuntze & $\begin{array}{l}\text { ACC } 1896 \text { \& AFC; AFC et al. 1786; } \\
\text { LMVP } 618\end{array}$ & T/A & \\
\hline $\mathbf{h}$ & Tristachya avenacea (Presl.) Scribn \& Merr. & $\begin{array}{l}\text { ACC 2192; ACC } 2341 \text { \& JGG; AFC et al. } \\
\text { 1787; JGG } 1093\end{array}$ & T/A & \\
\hline $\mathbf{h}$ & Tripsacum dactyloides (L.) L. & ACC 2333 \& JGG; AFC et al. 1788 & T/A & \\
\hline $\mathbf{h}$ & $\begin{array}{l}\text { Trisetum mexicanum (Swallen) S.D.Koch } \\
\text { Themidaceae }\end{array}$ & CLDL 3750 & T/A & \\
\hline $\mathbf{h}$ & $\begin{array}{l}\text { Bessera elegans Schult. f. } \\
\text { MAGNOLIOPSIDA } \\
\text { Acanthaceae }\end{array}$ & ACC 2166 \& JGG & T/A & \\
\hline $\mathbf{h}$ & Dyschoriste jaliscensis Kobuski & $\begin{array}{l}\text { ACC et al. 2209a; ACC } 2351 \text { \& JGG; } \\
\text { JGG } 1091\end{array}$ & T/A & \\
\hline $\mathbf{h}$ & Elytraria imbricata (Vahl) Pers. & $\begin{array}{l}\text { JGG et al. 193; AFC } 1857 \text { \& JGG; LMVP } \\
\text { 2085, 1355, 4734 }\end{array}$ & T/A & \\
\hline $\mathbf{h}$ & $\begin{array}{l}\text { Pseudoranthemum praecox (Benth.) Leonard } \\
\text { Amaranthaceae }\end{array}$ & ACC $2139 \&$ AFC & T/A & \\
\hline $\mathbf{h}$ & Iresine diffusa Humbl. \& Bonpl. ex Willd. & $\begin{array}{l}\text { ACC } 2357 \text { \& JGG; JGG et al. 906; } \\
\text { LMVP } 4743\end{array}$ & T/A & \\
\hline & Anacardiaceae & & & \\
\hline b & $\begin{array}{l}\text { Toxicodendron radicans (L.) Kuntze } \\
\text { Apiaceae }\end{array}$ & ACC 2334 \& JGG & T/A & \\
\hline $\mathbf{h}$ & $\begin{array}{l}\text { Arracacia tolucensis (Kunth) Hemsl. var. multifida } \\
\text { (S.Watson) Mathias \& Constance }\end{array}$ & ACC 2117 \& JGG; ACC et al. 2202 & T/A & \\
\hline $\mathbf{h}$ & $\begin{array}{l}\text { Donnellsmithia juncea (Humb. \& Bonpl. ex Spreng.) } \\
\text { Mathias \& Constance }\end{array}$ & ACC 2167 \& JGG; LMVP 1522 & T/A & \\
\hline $\mathbf{h}$ & Eryngium beecheyanum Hook. f. \& Arn. & LMVP 8264 & T/A & \\
\hline $\mathbf{h}$ & E. columnare Hemsl. & ACC 2112 \& JGG & T/A & \\
\hline $\mathbf{h}$ & E. palmeri Hemsl. & LMVP 2532 & T/A & \\
\hline $\mathbf{h}$ & $\begin{array}{l}\text { E. pectinatum C.Presl. ex DC. } \\
\text { Apocynaceae }\end{array}$ & LMVP 2375 & T/A & \\
\hline $\mathbf{h}$ & Asclepias auriculata Kunth & ACC et al. 2184 & T/A & \\
\hline $\mathbf{h}$ & A. contrayerba Sessé \& Moc. & ACC 2158 \& JGG; AFC et al. 1773 & T/A & \\
\hline
\end{tabular}


Apéndice 1. Continuación

\begin{tabular}{|c|c|c|c|c|}
\hline A & Especies & Cols./números de colecta & $\mathrm{H} / \mathrm{N}$ & NOM \\
\hline $\mathbf{h}$ & A. fournieri Wood. & CGR s.n. & T/A & \\
\hline $\mathbf{h}$ & A. glaucescens Kunth & JGG et al. 190 & T/A & \\
\hline $\mathbf{h}$ & A. ovata M.Martens \& Galeotti & ACC 1898a \& AFC & T/A & \\
\hline $\mathbf{h}$ & Macrosiphonia hypoleuca (Benth.) Muell.Arg. & ACC $2162 \&$ JGG & T/A & \\
\hline b & $\begin{array}{l}\text { Matelea sepicola W.D.Stevens } \\
\text { Araliaceae }\end{array}$ & AFC et al. 1763 & T/A & \\
\hline a & $\begin{array}{l}\text { Aralia humilis Cav. } \\
\text { Aristolochiaceae }\end{array}$ & JGG et al. 909 & T/A & \\
\hline \multirow[t]{2}{*}{ b } & Aristolochia pringlei Rose & $\begin{array}{l}\text { AFC et al. 1759; LMVP 1516, 14580; } \\
\text { LMVP et al. } 64\end{array}$ & T/A & \\
\hline & Asteraceae & & & \\
\hline $\mathbf{h}$ & Acourtia arachnolepis (B.L.Rob.) B.L.Rob & ACC $2137 \&$ AFC & T/A & \\
\hline $\mathbf{h}$ & $\begin{array}{l}\text { A. wislizeni (A.Gray) Reveal \& R.M.King var. } \\
\text { megacephala (A.Gray) Reveal \& R.M.King }\end{array}$ & DRS sn & T/A & \\
\hline $\mathbf{h}$ & Ageratella microphylla (Sch. Bip.) A.Gray ex S.Watson & $\begin{array}{l}\text { ACC } 1886 \text { \& AFC; LMVP 2077, 5105, } \\
4589 ; \text { MHS } 2497\end{array}$ & T/A & \\
\hline $\mathbf{h}$ & Ageratina calaminthifolia (Kunth) R.M.King \& H.Rob & ACC et al. 2206 & T/A & \\
\hline $\mathbf{h}$ & A. leptodictyon (A.Gray) R.M.King \& H.Rob & ACC 2156 \& JGG & T/A & \\
\hline $\mathbf{h}$ & A. muelleri (Sch. Bip. ex Klaff) R.M.King \& H.Rob. & ACC 1898 \& AFC & T/A & \\
\hline $\mathbf{h}$ & A. sp. 1 & ACC 2342 \& JGG & T/A & \\
\hline $\mathbf{r}$ & Ageratum corymbosum Zuccagni & $\begin{array}{l}\text { ACC } 1876 \text { \& AFC; ACC et al. 2191; } \\
\text { AFC et al. 1806; LMVP } 4191\end{array}$ & T/A & \\
\hline $\mathbf{h}$ & $\begin{array}{l}\text { Alloispermum palmeri (S.Watson ex A.Gray) Fernández } \\
\text { \& Urbatsch var. palmeri }\end{array}$ & ACC $2155 \&$ JGG & T/A & \\
\hline $\mathbf{h}$ & A. scabrifoluim (Hook. \& Arn.) H.Rob. & ACC et al. 2321 & T/A & \\
\hline $\mathbf{r}$ & Baccharis glutinosa Pers. & CGR s. n. & T/A & \\
\hline $\mathbf{r}$ & B. occidentalis S.F.Blake & ACC 2154 \& JGG; LMVP 4065 & T/A & \\
\hline $\mathbf{r}$ & B. pteronioides DC. & LMVP 4740, 4084 & T/A & \\
\hline h & Bidens odorata Cav. & AFC 1853 \& JGG, JGG et al. 171 & T/A & \\
\hline $\mathbf{h}$ & B. rostrata Melchert & $\begin{array}{l}\text { ACC } 1875 \text { \& AFC; AFC } 1821 \text { \& JGG; } \\
\text { ARC 5740; LMVP 4740; MHS } 2496\end{array}$ & T/A & \\
\hline $\mathbf{h}$ & Bolanosa coulteri A.Gray & AFC 1814 \& JGG; & T/A & \\
\hline h & Brickellia adenolepis (B.L.Rob.) Shinners & JGG et al. 903; ACC 2339 \& JGG & T/A & \\
\hline $\mathbf{h}$ & B. cuspidata A.Gray & $\begin{array}{l}\text { ACC } 1894 \text { \& AFC; AFC } 1813 \text { \& JGG; AFC } \\
\text { et al. 1789; MHS 2499; LMVP 1169, } 5107\end{array}$ & T/A & \\
\hline $\mathbf{h}$ & B. jaliscensis McVaugh & AFC 1812 \& JGG; MHS 2498; LMVP 8259 & T/A & \\
\hline $\mathbf{h}$ & Calea ternifolia Kunth & $\begin{array}{l}\text { ACC 1872, } 1882 \text { \& AFC; JGG et al. 184; } \\
\text { LMVP 2098, } 4593\end{array}$ & T/A & \\
\hline $\mathbf{h}$ & Coreopsis cuneifolia Green. & $\begin{array}{l}\text { ACC } 1842 \text { \& ARC; AFC et al. 1790; ARC } \\
\text { 5738; LMVP } 4595\end{array}$ & T/A & \\
\hline $\mathbf{h}$ & C. cyclocarpa S.F.Blaket & MHS 2500 & T/A & \\
\hline $\mathbf{r}$ & C. petrophila A.Gray \& S.Watson & $\begin{array}{l}\text { ACC } 1846 \text { \& ARC; AFC et al. 1783; } \\
\text { LMVP } 4588\end{array}$ & T/A & \\
\hline $\mathbf{r}$ & Cosmos landii Sherff var. achalconensis Melchertt & ACC 1869, 1880 \& AFC; AFC et al. 1787a & T/A & \\
\hline $\mathbf{h}$ & C. sulphureus Cav. & ACC 1880 \& AFC & T/A & \\
\hline $\mathbf{r}$ & Critoniopsis foliosa (Benth.) H.Rob & ACC et al. 2327; JGG et al. 180 & T/A & \\
\hline $\mathbf{h}$ & Dahlia coccinea Cav. & LMVP 4559 & T/A & \\
\hline h & D. pugana Aaron Rodr. \& A.Castro*† & $\begin{array}{l}\text { ACC } 1870 \text { \& AFC; ACC } 1847 \text { \& ARC; } \\
\text { ACC \& JGG 2177; ACC et al. 2209; AFC } \\
\text { et al. 1760, } 1869\end{array}$ & T/A & \\
\hline
\end{tabular}


Apéndice 1. Continuación

\begin{tabular}{|c|c|c|c|c|}
\hline A & Especies & Cols./números de colecta & $\mathrm{H} / \mathrm{N}$ & NOM \\
\hline h & $\begin{array}{l}\text { Dyssodia porophyllum (Cav.) Cav. var. cancellata } \\
\text { (Cass.) Strother }\end{array}$ & GNH et al. 22; LMVP 7124 & T/A & \\
\hline $\mathbf{h}$ & Erigeron exilis A.Gray & ACC 2336 \& JGG & T/A & \\
\hline h & Gamochaeta americana (Mill.) Wedd. & AFC et al. 1874 & T/A & \\
\hline $\mathbf{r}$ & Guardiola mexicana Bonpl. & JRR 20271; LMVP 4089 & T/A & \\
\hline h & lostephane heterophylla (Cav.) Hemsl. & ACC et al. 2186; AFC et al. 1778 & T/A & \\
\hline $\mathbf{h}$ & Lagascea helianthifolia Kunth var. levior (B.L.Rob.) B.L.Rob. & ACC et al. 2320 & T/A & \\
\hline h & Lasianthaea aurea (D.Don) K.M.Becker & AFC 1819 \& JGG; LMVP 1317 & T/A & \\
\hline h & L. palmeri (Greenm.) K.M.Beker & AFC et al. 1866 & T/A & \\
\hline $\mathbf{r}$ & Montanoa karvinskii (DC.) Sch.Bip. ex K.Koch & $\begin{array}{l}\text { ACC } 1881 \text { \& AFC; AFC et al. 1767; } \\
\text { LMVP } 2069\end{array}$ & T/A & \\
\hline h & Odontotrichum platylepis (B.L.Rob. \& Seaton) Rydb. & JGG et al. 172 & T/A & \\
\hline h & Olivaea tricuspis Sch.Bip. & CLDL 4353 & T/A & \\
\hline h & Pectis diffusa Hook. \& Arn. & LMVP 2084 & T/A & \\
\hline h & $\begin{array}{l}\text { Perymenium buphthalmoides DC. var. occidentale } \\
\text { McVaugh }\end{array}$ & LMVP 4074, 6435 & T/A & \\
\hline h & P. jaliscence B.L.Rob \& Greenm. var. latifolium McVaugh & LMVP 2037 & T/A & \\
\hline h & Perityle jaliscana A.Gray† & ACC 2440 \& RRM & T/A & \\
\hline h & Porophyllum lindenii Sch.Bip. & JGG et al. 908 & T/A & \\
\hline h & Psacalium poculiferum (S.Watson) Rydb. & ACC 2176 \& JGG; LMVP 4073 & T/A & \\
\hline h & Roldana sessilifolia (Hook. \& Arn.) H.Rob. \& Brettell & JGG et al. 188 & T/A & \\
\hline h & $\begin{array}{l}\text { Schkuhria pinnata (Lam.) Kuntze ex Thell. var. virgata } \\
\text { (Llave) Heiser }\end{array}$ & CLDL 377; LMVP 4606, 8265, 14563 & T/A & \\
\hline $\mathbf{r}$ & Stevia jaliscensis B.L.Rob. & LMVP 4738, 7084 & T/A & \\
\hline $\mathbf{h}$ & S. ovalis (B.L.Rob.) B.L.Rob.† & $\begin{array}{l}\text { ACC et al. 2196; AFC et al. 1807; } \\
\text { LMVP 4584, } 14561\end{array}$ & T/A & \\
\hline $\mathbf{r}$ & S. viscida Kunth & $\begin{array}{l}\text { ACC } 1892 \text { \& AFC; AFC } 1817 \text { \& JGG; } \\
\text { AFC et al. 1793; JGG et al. 174, } 179\end{array}$ & T/A & \\
\hline $\mathbf{h}$ & S. sp. 1 & ACC 2094 \& JGG & T/A & \\
\hline $\mathbf{h}$ & Tagetes filifolia Lag. & JGG et al. 202 & T/A & \\
\hline $\mathbf{h}$ & T. subulata Cerv. & $\begin{array}{l}\text { ACC } 1888 \text { \& AFC; JGG et al. 197; } \\
\text { LMVP 1357; MHS } 2494\end{array}$ & T/A & \\
\hline $\mathbf{r}$ & Verbesina angustifolia (Benth.) Blake & ACC 1891 \& AFC; AFC 1846 \& JGG & T/A & \\
\hline $\mathbf{r}$ & V. cinerascens B.L.Rob. \& Greenm. & AFC $1815,1833 \&$ JGG & T/A & \\
\hline $\mathbf{h}$ & V. oxylepis Blake & ACC 2092, $2175 \&$ JGG & T/A & \\
\hline $\mathbf{h}$ & V. tequilana J.R.Coleman & ACC 1873 \& AFC; AFC et al. 1791 & T/A & \\
\hline $\mathbf{r}$ & Vernonanthura cordata (Kunth) H.Rob. & ACC et al. 2322 & T/A & \\
\hline $\mathbf{r}$ & Viguiera angustifolia (Hook. \& Arn.) S.F.Blake & ACC et al. 2325 & T/A & \\
\hline $\mathbf{h}$ & V. ensifolia (Sch. Bip.) S.F.Blake & ACC 2325 \& JGG & T/A & \\
\hline $\mathbf{r}$ & V. palmeri B.L.Rob. \& Greenm. & ACC et al. 2319 & T/A & \\
\hline $\mathbf{h}$ & V. schultzii S.F.Blake & AFC 1816 \& JGG & T/A & \\
\hline $\mathbf{h}$ & Wedelia grayi McVaught & $\begin{array}{l}\text { ACC } 2163 \text { \& JGG; AFC et al. 1789; } \\
\text { LMVP 4600; MHS } 2495\end{array}$ & T/A & \\
\hline $\mathbf{h}$ & $\begin{array}{l}\text { Zinnia angustifolia Kunth var.angustifolia } \\
\text { Begoniaceae }\end{array}$ & JGG et al. 196 & T/A & \\
\hline $\mathbf{h}$ & Begonia angustiloba A.DC. & JGG et al. 187 & T/A & \\
\hline $\mathbf{h}$ & B. gracilis Kunth & LMVP 4597 & T/A & \\
\hline $\mathbf{h}$ & $\begin{array}{l}\text { B. tapatia Burt-Utley \& McVaugh } \\
\text { Bignoniaceae }\end{array}$ & AFC et al. 1766, 1774; JGG et al. 188 & T/A & \\
\hline $\mathbf{a}$ & Tecoma stans (L.) Juss. ex Kunth & JGG 1197 & T/A & \\
\hline
\end{tabular}


Apéndice 1. Continuación

\begin{tabular}{|c|c|c|c|c|}
\hline A & Especies & Cols./números de colecta & $\mathrm{H} / \mathrm{N}$ & NOM \\
\hline & Boraginaceae & & & \\
\hline $\mathbf{h}$ & $\begin{array}{l}\text { Lennoa medreporoides Lex. } \\
\text { Buddleiaceae }\end{array}$ & AFC $1824 \&$ JGG & T/L & \\
\hline $\mathbf{h}$ & $\begin{array}{l}\text { Buddleia sessiliflora Kunth } \\
\text { Burseraceae }\end{array}$ & ACC 2347 \& JGG & T/A & \\
\hline $\mathbf{a}$ & Bursera fagaroides (Kunth) Engl. & LMVP 62 & $\mathbf{T} / \mathbf{A}$ & \\
\hline $\mathbf{a}$ & B. multijuga Engl. & AFC et al. 1881 & T/A & \\
\hline $\mathbf{a}$ & B. palmeri S.Watson & AFC 1751 \& EASM & T/A & \\
\hline a & $\begin{array}{l}\text { B. penicillata (DC.) Engl. } \\
\text { Cactaceae }\end{array}$ & LMVP et al. s.n. & T/A & \\
\hline $\mathbf{h}$ & Mammillaria jaliscana (Britton \& Rose) Boed. & AFC1846 \& JGG & $\mathbf{R} / \mathbf{A}$ & \\
\hline $\mathbf{r}$ & $\begin{array}{l}\text { Opuntia jaliscana Bravo } \\
\text { Campanulaceae }\end{array}$ & SZQ s. n. & T/A & \\
\hline $\mathbf{h}$ & Diastatea tenera (A.Gray) McVaugh & ACC $1874 \&$ AFC & T/A & \\
\hline $\mathbf{h}$ & $\begin{array}{l}\text { Lobelia laxiflora Kunth } \\
\text { Caryophyllaceae }\end{array}$ & ACC 2338 \& JGG & T/A & \\
\hline $\mathbf{h}$ & Drymaria villosa Schltdl. \& Cham. & $\begin{array}{l}\text { AFC et al. 1864; JGG 1095; LMVP 577, } \\
1315\end{array}$ & T/A & \\
\hline & Clethraceae & & & \\
\hline a & $\begin{array}{l}\text { Clethra rosei Britton } \\
\text { Cistaceae }\end{array}$ & ACC 2118 \& JGG; LMVP 2099, 5934 & T/A & \\
\hline $\mathbf{h}$ & Helianthemum patens Hemsl. & ACC $2128 \&$ AFC & T/A & \\
\hline $\mathbf{h}$ & $\begin{array}{l}\text { Lechea tripetala (Moc. \& Sessé ex Dunal) Britton } \\
\text { Convolvulaceae }\end{array}$ & LMVP 608 & $\mathbf{T} / \mathbf{A}$ & \\
\hline $\mathbf{h}$ & Evolvulus alsinoides L. & $\begin{array}{l}\text { ACC } 2136 \text { \& AFC; JGG et al. 176; } \\
\text { LMVP } 4095\end{array}$ & T/A & \\
\hline $\mathbf{h}$ & Ipomoea capillacea (Kunth) G.Don & $\begin{array}{l}\text { ACC } 2121 \text { \& JGG; ACC et al. 2207; } \\
\text { LMVP } 14575\end{array}$ & T/A & \\
\hline b & I. laeta A.Gray & $\begin{array}{l}\text { ACC } 2153 \text { \& JGG; ACC et al. 2182; } \\
\text { AFC et al. 1769; JGG et al. } 175\end{array}$ & T/A & \\
\hline b & I. orizabensis (Pelletan) Ledeb. ex Steud var. orizabensis & $\begin{array}{l}\text { ACC et al. } 2179 ; \text { JGG et al. 191; } \\
\text { LMVP } 14574\end{array}$ & T/A & \\
\hline $\mathbf{h}$ & $\begin{array}{l}\text { I. stans Cav. } \\
\text { Crassulaceae }\end{array}$ & LMVP 1348 & T/A & \\
\hline $\mathbf{h}$ & Sedum jaliscanum S.Watson & $\begin{array}{l}\text { AFC } 1823 \text { \& JGG; AFC et al. 1801; } \\
\text { MHS } 2487\end{array}$ & R/A & \\
\hline & Ericaceae & & & \\
\hline a & $\begin{array}{l}\text { Agarista mexicana (Hemsl.) Judd var. pinetorum } \\
\text { (Standl. \& L.O.Williams) Judd }\end{array}$ & $\begin{array}{l}\text { CLDL 87; LMVP 150, 531, 4082, } \\
5937,9360\end{array}$ & T/A & \\
\hline a & Bejaria aestuans Mutis ex L. & $\begin{array}{l}\text { ACC et al. } 2203 ; \text { AFC } 1748 \text { \& EASM; } \\
\text { LMVP } 5936\end{array}$ & T/A & \\
\hline $\mathbf{r}$ & Comarostaphylis glaucescens (Kunth) Zucc. ex Klotzsch & $\begin{array}{l}\text { ACC } 2165 \text { \& JGG; ACC } 2442 \text { \& RRM; } \\
\text { JGG et al. 904; LMVP 4580, } 5932\end{array}$ & T/A & \\
\hline $\mathbf{a}$ & Gaultheria hirtiflora Benth. & LMVP 2466 & T/A & \\
\hline $\mathbf{r}$ & $\begin{array}{l}\text { Vaccinium stenophyllum Steud. } \\
\text { Euphorbiaceae }\end{array}$ & ACC 2164 \& JGG; AFC et al. 1809 & T/A & \\
\hline $\mathbf{h}$ & Acalypha multispicata S.Watson & $\begin{array}{l}\text { ACC } 2150 \text { \& JGG; AFC et al. 1865, } \\
\text { LMVP } 4078\end{array}$ & T/A & \\
\hline $\mathbf{h}$ & Euphorbia guadalajarana S.Watson & $\begin{array}{l}\text { AFC et al. 1781; AFC } 1818 \text { \& JGG; } \\
\text { LMVP 5101, 5102 }\end{array}$ & T/A & \\
\hline
\end{tabular}


Apéndice 1. Continuación

\begin{tabular}{|c|c|c|c|c|}
\hline A & Especies & Cols./números de colecta & $\mathrm{H} / \mathrm{N}$ & NOM \\
\hline $\mathbf{h}$ & E. hyssopifolia L. & JGG et al. 204 & T/A & \\
\hline h & E. macropus (Klotzsch. \& Garcke) Boiss & $\begin{array}{l}\text { ACC et al. 2194; AFC } 1755 \text { \& EASM; } \\
\text { LMVP } 1610\end{array}$ & T/A & \\
\hline $\mathbf{h}$ & E. sphaerorhiza Benth. & $\begin{array}{l}\text { ACC } 2102 \text { \& JGG; JRR 20272; LMVP 4079; } \\
\text { MJCB et al. s. n. }\end{array}$ & T/A & \\
\hline $\mathbf{h}$ & E. subreniformis S.Watson & JGG et al. 178; LMVP 5090 & T/A & \\
\hline $\mathbf{r}$ & $\begin{array}{l}\text { Stillingia zelayensis (Kunth) Müll. Arg. } \\
\text { Fabaceae }\end{array}$ & LMVP 4090 & T/A & \\
\hline $\mathbf{a}$ & Acacia pennatula (Schltdl. \& Cham.) Benth. & JGG et al. 1192 & T/A & \\
\hline a & $\begin{array}{l}\text { Acaciella angustissima (Mill.) Britton \& Rose } \\
\text { var. angustissima }\end{array}$ & ACC 2358, 2174 \& JGG; JGG et al. 901 & T/A & \\
\hline a & A. tequilana (S.Watson) Britton \& Rose & ACC 2174; AFC et al. 1879; LMVP 4639 & T/A & \\
\hline $\mathbf{r}$ & Aeschynomene petraea B.L.Rob. & ACC 2138 \& AFC; JGG et al. 905 & T/A & \\
\hline $\mathbf{r}$ & Calliandra grandiflora (L'Her) Benth. & AFC et al. 1796; JGG et al. 898 & T/A & \\
\hline $\mathbf{r}$ & C. hirsuta (G.Don) Benth. & $\begin{array}{l}\text { ACC } 1848 \text { \& ARC; AFC et al. 1796a; } \\
\text { LMVP4599 }\end{array}$ & T/A & \\
\hline b & Canavalia villosa Benth. & $\begin{array}{l}\text { ACC et al. 2186; ACC } 2171 \text { \& JGG; } \\
\text { AFC et al. 1761, 1804; LMVP } 15636\end{array}$ & T/A & \\
\hline $\mathbf{h}$ & $\begin{array}{l}\text { Chamaecrista absus (L.) H.S.Irwin \& Barneby var. } \\
\text { meonandra (H.S.Irwin \& Barneby) H.S.Irwin \& Barneby }\end{array}$ & JGG et al. 185 & T/A & \\
\hline h & $\begin{array}{l}\text { C. nictitans Moench var. jaliscensis (Greenm.) } \\
\text { H.S.Irwin \& Barneby }\end{array}$ & JGG et al. 194 & T/A & \\
\hline $\mathbf{h}$ & C. rotundifolia (Pers.) Greene & JGG et al. 200 & T/A & \\
\hline $\mathbf{r}$ & $\begin{array}{l}\text { C. serpens (L.) Greene var. wrightii (A.Gray) } \\
\text { H.S.Irwin \& Barneby }\end{array}$ & $\begin{array}{l}\text { ACC et al. 2193; JGG et al. 173; LMVP } \\
\text { 2099, 4596; PFS } 1448\end{array}$ & T/A & \\
\hline h & Clitoria triflora S.Watson & $\begin{array}{l}\text { ACC } 1884 \text { \& AFC; ACC } 2170 \text { \& JGG; } \\
\text { AFC et al. 1877; ARC } 450\end{array}$ & T/A & \\
\hline b & Cologania angustifolia Kunth & ACC $2101 \&$ JGG & T/A & \\
\hline b & C. broussoneti (Balb.) DC. & AFC et al. 1795 & T/A & \\
\hline b & C. procumbens Kunth & LMVP 4067 & T/A & \\
\hline h & Crotalaria quercetorum Brandegee & JGG et al. 203, LMVP s.n. & T/A & \\
\hline $\mathbf{h}$ & C. sagittalis $\mathrm{L}$. & AFC et al. 1886 & T/A & \\
\hline h & Dalea cliffortiana Willd. & LMVP 8257 & T/A & \\
\hline h & D. polystachya (Sessé \& Moc.) Barneby & LMVP 3524 & T/A & \\
\hline $\mathbf{h}$ & D. revoluta S.Watson & LMVP s.n. & T/A & \\
\hline h & D. sericea Lag. & LMVP 3776 & T/A & \\
\hline $\mathbf{h}$ & D. tomentosa (Cav.) Willd. & $\begin{array}{l}\text { ACC } 1895 \text { \& AFC; AFC } 1839 \text { \& JGG; } \\
\text { LMVP } 5104\end{array}$ & T/A & \\
\hline $\mathbf{h}$ & D. versicolor Zucc. & JGG et al. 900 & T/A & \\
\hline $\mathbf{r}$ & Desmodium angustifolium (Kunth) DC. & CLDL 253; LMVP 2079, 4592, 14567 & T/A & \\
\hline $\mathbf{h}$ & D. aparines (Link.) DC. & AFC et al. 1798 & T/A & \\
\hline $\mathbf{r}$ & D. jaliscanum S.Watson & AFC s. n. \& JGG. & T/A & \\
\hline $\mathbf{h}$ & D. macrostachyum Hemsl. & AFC 1838 \& JGG & T/A & \\
\hline $\mathbf{r}$ & $\begin{array}{l}\text { D. orbiculare Schltdl. var. rubricaule (Rose \& Painter) } \\
\text { B.G.Schub. \& McVaugh }\end{array}$ & $\begin{array}{l}\text { ACC } 1897 \text { \& AFC; AFC } 1805 \text { \& JGG; } \\
\text { LMVP 1612, } 4594\end{array}$ & T/A & \\
\hline $\mathbf{r}$ & D. plicatum Schltdl. \& Cham. & ACC 2134 \& AFC; JGG et al. 896 & T/A & \\
\hline h & D. procumbens (Mill.) Hitchc. & LMVP 2071 & T/A & \\
\hline h & D. scorpiurus (Sw.) Desv. & LMVP 4746 & T/A & \\
\hline h & D. sericophyllum Schltdl. & CLDL 356; LMVP 2101 & T/A & \\
\hline $\mathbf{h}$ & D. volubile (Schindl.) B.G.Schub. \& McVaugh & Schubert s.n. & T/A & \\
\hline
\end{tabular}


Apéndice 1. Continuación

\begin{tabular}{|c|c|c|c|c|}
\hline A & Especies & Cols./números de colecta & $\mathrm{H} / \mathrm{N}$ & NOM \\
\hline $\mathbf{a}$ & Diphysa suberosa S.Watson & JAPR 1002 & T/A & \\
\hline $\mathbf{r}$ & D. thurberi (A.Gray) Rydb. ex Standl. & ACC 2173 \& JGG; ACC et al. 2200 & T/A & \\
\hline $\mathbf{r}$ & Eriosema diffusum (Kunth) G.Don & $\begin{array}{l}\text { ACC } 1887 \text { \& AFC; AFC et al. 1802; } \\
\text { LMVP } 5099\end{array}$ & $\mathbf{T} / \mathbf{A}$ & \\
\hline $\mathbf{r}$ & E. grandiflorum (Scltdl. \& Cham.) G.Don & AFC et al. 1792; LMVP 4583, 5108 & T/A & \\
\hline $\mathbf{r}$ & E. pulchellum (Kuth) G.Don & ACC 2093 \& JGG & T/A & \\
\hline $\mathbf{r}$ & Erythrina montana Rose \& Standl. & ACC 2157 \& JGG & T/A & \\
\hline $\mathbf{r}$ & Indigofera densiflora M.Martens \& Galeotti & AFC et al. 1794, 1889; MHS 2491 & T/A & \\
\hline $\mathbf{a}$ & Leucaena macrophylla Benth. & ACC 2087 \& JGG & T/A & \\
\hline a & Lysiloma acapulcense (Kunth) Benth. & ACC 2140 \& AFC; LMVP 4596. & T/A & \\
\hline b & Macroptilium gibbosifolium (Ort.) A.Delgado & AFC et al. 1797; JGG et al. 192 & T/A & \\
\hline $\mathbf{r}$ & Marina crenulata (Hook. \& Arn.) Barneby & JGG et al. 897 & T/A & \\
\hline b & $\begin{array}{l}\text { Mimosa albida Humb. \& Bonpl. ex Willd. var. strigosa } \\
\text { (Willd.) B.L.Rob }\end{array}$ & CSR 27 & T/A & \\
\hline h & M. pudica L. & LMVP 4601 & T/A & \\
\hline h & M. quadrivalvis L. & ACC 2125 \& JGG & T/A & \\
\hline h & M. tequilana S.Watson & LMVP 2078, 7026 & T/A & \\
\hline b & Phaseolus jaliscanus Piper & ACC 2181 & T/A & \\
\hline b & P. maculatus Scheele & AFC et al. 1800 & T/A & \\
\hline b & P. pauciflorus Sessé \& Moc. ex G.Don & AFC et al. 1888 & T/A & \\
\hline b & Rhynchosia precatoria (Humb. \& Bonpl. ex Willd) DC. & $\begin{array}{l}\text { ACC et al. 2318a; AFC } 1843 \text { \& JGG; } \\
\text { ARC } 5735\end{array}$ & T/A & \\
\hline a & Senna didymobotrya (Fresen) H.S.Irwin \& Barneby & GL s.n & T/A & \\
\hline $\mathbf{r}$ & Tephrosia nicaraguensis Oerst. & ACC 2172 \& JGG; AFC et al. 1799 & T/A & \\
\hline $\mathbf{h}$ & T. watsoniana (Standl.) J.F.Macbr. & ACC 2126 \& JGG; LMVP 4666, 4640 & T/A & \\
\hline $\mathbf{h}$ & $\begin{array}{l}\text { Zornia reticulata Sm. } \\
\text { Fagaceae }\end{array}$ & ACC et al. 2180; JGG et al. 201 & T/A & \\
\hline a & Quercus candicans Née & SJC s.n. & T/A & \\
\hline $\mathbf{a}$ & Q. castanea Née & LMVP 4715 & T/A & \\
\hline $\mathbf{a}$ & Q. castanea x obtusata & ACC $2161 \&$ JGG & T/A & \\
\hline a & Q. cocolobifolia Trel. & ACC 2114 \& JGG & T/A & \\
\hline a & Q. conspersa Benth. & ACC 2438 \& RRM; AFC et al. 1890 & T/A & \\
\hline $\mathbf{a}$ & Q. convallata Trel. & AFC 1747 \& EASM & T/A & \\
\hline $\mathbf{a}$ & Q. magnoliifolia Née & JGG et al. 1193 & T/A & \\
\hline a & Q. praineana Trel. & $\begin{array}{l}\text { ACC } 2439 \text { \& RRM; ACC et al. 2195; } \\
\text { AFC } 1752 \text { \& EASM; LMVP } 5931\end{array}$ & T/A & \\
\hline $\mathbf{a}$ & Q. resinosa Liebm. & $\begin{array}{l}\text { ACC } 2146 \text { \& AFC; ACC } 2113 \text { \& JGG; } \\
\text { LMVP 6439, 6441, } 9546\end{array}$ & T/A & \\
\hline $\mathbf{a}$ & Q. rugosa Née & JGG et al. 1194 & T/A & \\
\hline \multirow[t]{2}{*}{$\mathbf{a}$} & Q. viminea Trel. & $\begin{array}{l}\text { ACC } 2147 \text { \& AFC; AFC et al. 1779; } \\
\text { LMVP 4062, 4702, 4708, 4762, } 6969\end{array}$ & T/A & \\
\hline & Gentianaceae & & & \\
\hline $\mathbf{h}$ & Gyrandra sp. & AFC 1830 \& JGG & T/A & \\
\hline h & $\begin{array}{l}\text { Zeltnera quitensis (Kunth) G.Mans. } \\
\text { Gesneriaceae }\end{array}$ & AFC 1887 et al. & T/A & \\
\hline $\mathbf{r}$ & $\begin{array}{l}\text { Moussonia elegans Decne. } \\
\text { Hypericaceae }\end{array}$ & ACC 2335 \& JGG & T/A & \\
\hline h & $\begin{array}{l}\text { Hypericum moranense Kunth } \\
\text { Lamiaceae }\end{array}$ & LMVP 13176 & T/A & \\
\hline $\mathbf{r}$ & Asterohyptis stellulata (Benth) Epling & AFC 1859 \& JGG; LMVP 4736 & T/A & \\
\hline
\end{tabular}


Apéndice 1. Continuación

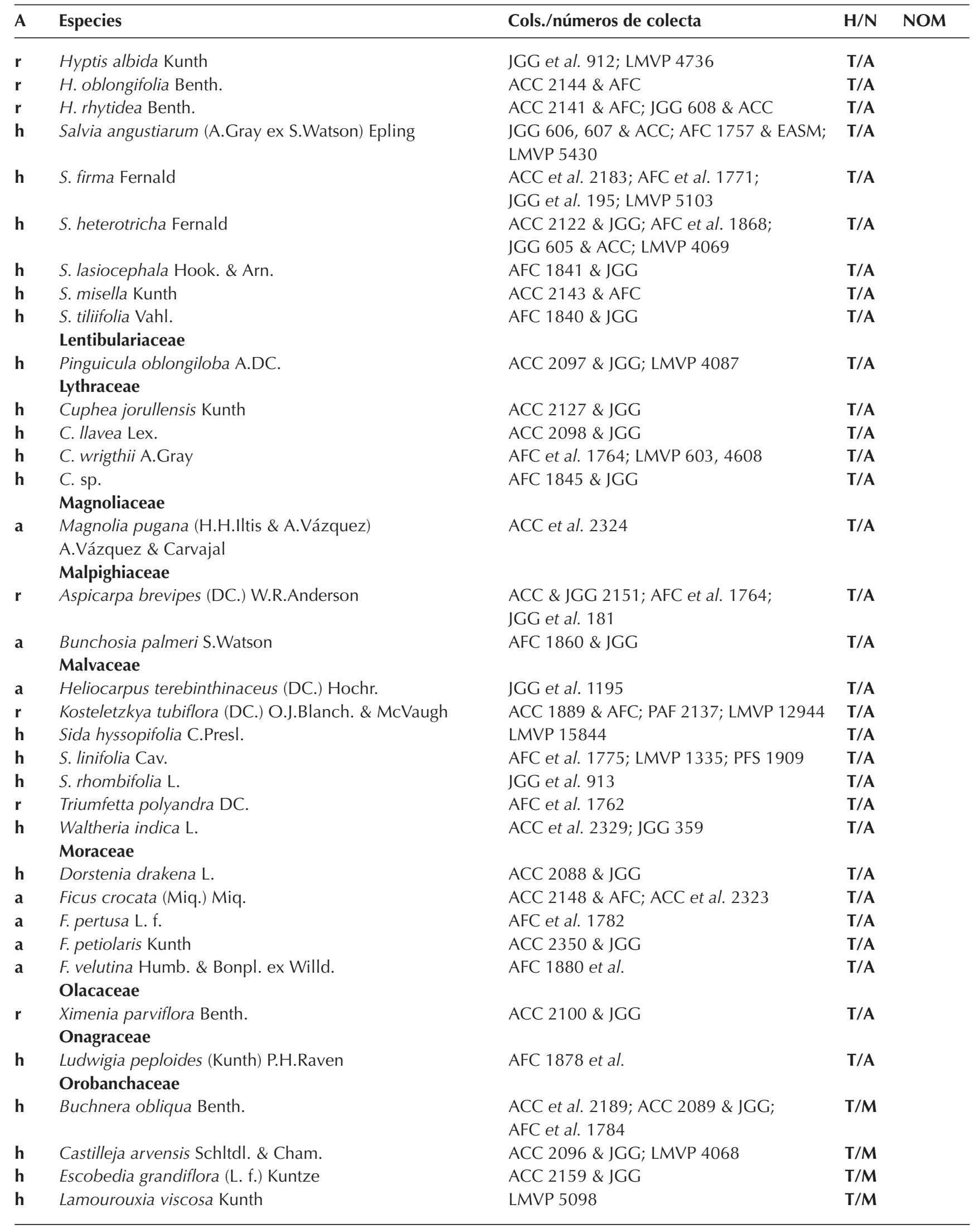


Apéndice 1. Continuación

\begin{tabular}{|c|c|c|c|c|}
\hline A & Especies & Cols./números de colecta & $\mathrm{H} / \mathrm{N}$ & NOM \\
\hline h & Oxalis hernandezii DC. & ACC 2085 \& JGG & $\mathrm{T} / \mathrm{A}$ & \\
\hline \multirow[t]{2}{*}{ b } & Passiflora foetida L. & AFC 1855 \& JGG & T/A & \\
\hline & Piperacerae & & & \\
\hline h & Scoparia dulcis L. & LMVP 4590 & $\mathbf{T} / \mathbf{A}$ & \\
\hline \multirow[t]{2}{*}{ h } & Stemodia durantifolia (L.) Sw. & LMVP 406, 1604 & T/A & \\
\hline & Polemoniaceae & & & \\
\hline \multirow[t]{2}{*}{$\mathbf{r}$} & Loeselia mexicana (Lam.) Brand & ACC 2349 \& JGG & T/A & \\
\hline & Polygalaceae & & & \\
\hline h & Ranunculaceae & & & \\
\hline \multirow[t]{2}{*}{ b } & Clematis dioica L. & ACC 2356 \& JGG; JGG et al. 914 & T/A & \\
\hline & Rhamnaceae & & & \\
\hline \multirow[t]{2}{*}{$\mathbf{a}$} & Colubrina greggii S.Watson & ACC $2135 \&$ AFC & T/A & \\
\hline & Rubiaceae & & & \\
\hline $\mathbf{r}$ & Bouvardia terniflora (Cav.) Schltdl. & ACC 1878 \& AFC; ACC et al. 2340 & T/A & \\
\hline h & Gallium mexicanum Kunth & JGG et al. 910 & $\mathbf{T} / \mathbf{A}$ & \\
\hline h & Mitracarpus hirtus (Lam.) DC. & $\begin{array}{l}\text { ACC et al. 2190; AFC et al. 1765; } \\
\text { JGG } 1090\end{array}$ & T/A & \\
\hline $\mathbf{a}$ & Randia capitata DC. & ACC $2119 \&$ JGG & T/A & \\
\hline $\mathbf{r}$ & $\begin{array}{l}\text { S. lycopersicum L. var. cerasiforme (Dunal) D.M.Spooner, } \\
\text { J.Anderson \& R.K.Jansen }\end{array}$ & ARC 5032 & T/A & \\
\hline $\mathbf{r}$ & S. pseudocapsicum L. & JBG s. n. & $\mathrm{T} / \mathrm{A}$ & \\
\hline h & S. stoloniferum Schltdl. & AFC et al. 1883; DMS et al. 4086 & T/A & \\
\hline \multirow[t]{2}{*}{$\mathbf{r}$} & S. umbellatum Miller & AFC 1752 \& EASM & T/A & \\
\hline & Styracaceae & & & \\
\hline \multirow[t]{3}{*}{$\mathbf{r}$} & Styrax jaliscana S.Watson & ACC 1893 \& AFC; AFC 1750 \& EASM; & T/A & \\
\hline & & LMVP 4585; MHS 2489 & & \\
\hline & Verbenaceae & & & \\
\hline $\mathbf{a}$ & Lippia umbellata Cav. & AFC 1861 \& JGG; JGG et al. 911 & T/A & \\
\hline \multirow[t]{2}{*}{ h } & Priva aspera Kunth & AFC et al. 1871 & T/A & \\
\hline & Violaceae & & & \\
\hline \multirow[t]{2}{*}{$\mathbf{h}$} & Viola oxyodontis Ballard & ACC 2115 \& JGG; AFC 1858 \& JGG & T/A & \\
\hline & Zygophyllaceae & & & \\
\hline h & Kallstroemia grandiflora Torrey ex A.Gray & AFC 1737 \& JGG & T/A & \\
\hline
\end{tabular}

